\title{
Funzioni continue da una parte con particolare riguardo alla loro derivabilità unilaterale.
}

\author{
Memoria di T. Viola (a Bologna).
}

Sunto. - Si estende alle funzioni contimue da una parte un teorema fondamentale di DENJOY sui numeri derivati e se ne traggono notevoli conseguenze. Si dimostra che l'aggregato dei punti di discontinuità ̀̀ numerabile, che sotto determinate condiaioni può essere denso e in quest'ultina ipotesi se ne studia la struttura. Si dànno infine degli esempi di funzioni continue verso destra.

In una piccola Nota comparsa nel $n .^{\circ} 1$, amo $\mathrm{X}(1931)$, del * Bollettino dell'Unione Matematica Italiana col titolo Sulle funzioni continue da una parte e sulla derivazione unilaterale, ho promesso di pubblicare per esteso la mia tesi di laurea, presentata nell' autunno 1930 alla Regia Università di Bologna. Mantengo ora la promessa per la prima parte della mia tesi ove trovansi dimostrate le proposizioni enunciate ai $n .^{i} 2,3$, di quella Nota.

Rivolgo un pensiero di devota riconoscenza al mio Maestro Beppo Levi che con tanta intelligenza e con tanto amore mi ha assistito in questa fatica.

\section{§ 1. Estensione di un teorema fondamentale di Denjoy.}

1. Definizioni. - Una funzione $F(x)$ della variabile reale $x$, data in un intervallo $\overline{a b}$, ̀े continua verso destra in un punto $x_{0}$ di $\overline{a b}$ se, assegnato comunque un numero $\varepsilon>0$, esiste sempre un numero $\delta>0$ tale che, se $0<x-x_{0}<\delta$, ̀े $\left|F(x)-F\left(x_{0}\right)\right|<\varepsilon$.

Per una funzione $F(x)$ continua verso destra in un punto $x_{0}$ seguirò la terminologia abituale ed adotterò notazioni in uso presso varí autori. Diró dunque:

«Rapporto incrementale destro» di $F(x)$ in $x_{0}$ il rapporto

$$
\frac{F(x)-F\left(x_{0}\right)}{x-x_{0}}
$$

per $x>x_{0}$ e sufficientemente prossimo ad $x_{0}$; 
"Numero derivato superiore destro» di $F(x)$ in $x_{0}$ il numero

$$
\bar{D}_{+} F\left(x_{0}\right)=\operatorname{limite~massimo~}_{x \rightarrow x_{0}+0} \frac{F(x)-F\left(x_{0}\right)}{x-x_{0}},
$$

cioè il massimo dei valori limiti del rapporto incrementale destro di $F(x)$ in $x_{0}$ quando $x$ tende a $x_{0}$; analogamente

"Numero derivato inferiore destro * di $F(x)$ in $x_{0}$ il numero

$$
\underline{D}_{+} F\left(x_{0}\right)=\underset{x \rightarrow x_{0}+0}{\operatorname{limite} \operatorname{minim} o} \frac{F(x)-F\left(x_{0}\right)}{x-x_{0}}
$$

cioè il minimo dei valori limiti del rapporto inerementale destro di $F(x)$ in $x_{0}$ quando $x$ tende a $x_{0}$.

Se una funzione $F(x)$ è continua verso destra in tutto un intervallo $\overline{a b}$ (cioè in tutti i punti di un intervallo $\overline{a b}$ ), si puó immaginare $x_{0}$ variabile in $\overline{a b}$ (dopochè per ogni suo valore siano supposte effettuate le dette operazioni di limite) e, sostituendogli a sua volta per semplicita e chiarezza il simbolo di variabile indipendente $x$, si viene alla considerazione delle funzioni

$$
\bar{D}_{+} F(x), \quad \underline{D}_{+} F(x)
$$

dette rispettivamente "Derivata superiore destra * $e$ "Derivata interiore destra» di $F(x)$. Si ha, per ogni valore di $x$,

$$
\bar{D}_{+} F(x) \geq \underline{D}_{+} F(x) \text {. }
$$

Se, per un valore $x_{0}$ di $x$, e $\bar{D}_{+} F\left(x_{0}\right)=D_{+} F\left(x_{0}\right)$, la funzione $F(x)$ dicesi * derivabile verso destra" in $x_{0}$ e si scrive $\bar{D}_{+} F\left(x_{0}\right)=\underline{D}_{+} F\left(x_{0}\right)=D_{+} F\left(x_{0}\right)$. In tal caso è

$$
D_{+} F\left(x_{0}\right)=\lim _{x \rightarrow x_{0}+0} \frac{F(x)-F\left(x_{0}\right)}{x-x_{0}} .
$$

Se ciò avviene in tutto $\overline{a b}$, resta definita la fnnzione $D_{+} F(x)$ "Derivata destra $»$ di $F(x)$.

Analoghe definizioni valgono per una funzione continua verso sinistra.

Se, per un valore $x_{0}$ di $x$, è $\bar{D}_{+} F\left(x_{0}\right)=D_{+} F\left(x_{0}\right)=\bar{D}_{-} F\left(x_{0}\right)=D_{-} F\left(x_{0}\right)$, la funzione $F(x)$ dicesi «derivabile» in $x_{0}$ e si scrive

$$
\begin{gathered}
\bar{D}_{+} F\left(x_{0}\right)=D_{+} F\left(x_{0}\right)=D_{+} F\left(x_{0}\right)= \\
=\bar{D}_{-} F^{\prime}\left(x_{0}\right)=D_{-} F\left(x_{0}\right)=D_{-} F\left(x_{0}\right)=D F\left(x_{0}\right)=F^{\prime}\left(x_{0}\right) .
\end{gathered}
$$


In tal caso è

$$
D F\left(x_{0}\right)=F^{\prime}\left(x_{0}\right)=\lim _{x \rightarrow x_{0}} \frac{F(x)-F\left(x_{0}\right)}{x-x_{0}} .
$$

Se ciò avviene in tutto $\overline{a b}$, resta definita la funzione $D F(x)=F^{\prime}(x)$ * derivata» di $F(x)$.

Io considerero, sempre nel presente lavoro, generiche funzioni $F(x)$ continue da una stessa parte in tutto un intervallo $\bar{a} \bar{b}$. Per fissare le idee supporrò sempre che la continuita in tutto l'intervallo $\overline{a b}$ sussista dalla parte destra.

2. Si può estendere, coi dovuti accorgimenti, ad una funzione $F(x)$ ovunque continua verso destra in $\overline{a b}$ il teor. fondamentale di DenJox sui numeri derivati delle funzioni continue e da lui chiamato "primo teorema" o "teorema descrittivo $\times\left({ }^{1}\right)$. Ne dedurrò delle applicazioni analoghe ad applicazioni dedotte dal Denjoy per le funzioni continue. Riporto le dimostrazioni del Denjox punto per punto, wa con le necessarie leggere varianti.

TeOREma fondamentale. Se esiste un'infinita d'intervalli

$$
S_{1}, S_{2}, S_{3}, \ldots, S_{n}, \ldots
$$

$\left(S_{n} \equiv \overline{c_{n} a_{n}}\right)$, la cui lunghezza tende a 0 per $n$ tendente ad $\infty$, i cui estremi sinistri $c_{1}, c_{2}, \ldots, c_{n}, \ldots$ formano un insieme ovunque denso in $\overline{a b}$; se, d'altra parte, il rapporto incrementale $l_{n}$ della funzione $F(x)$ su $S_{n}$

a) resta costantemente superiore a un numero fisso $k$,

b) oppure resta costantemente inferiore a un numero fisso $k$,

c) oppure tende verso un limite unico finito od infinito $l$,

allora, indicato con $E$ l'insieme dei punti di $\overline{a b}$ in cui è rispettivamente

a) $\bar{D}_{+} F(x) \geq k$

b) oppure $D_{+} F(x) \leq k$,

c) oppure il numero $l$ è un derivato mediano $\left({ }^{2}\right)$ od estremo destro,

l'insieme $E$ cosl definto è ovunque denso in $\overline{a b}$ ed anzi è un residuale $\left({ }^{3}\right)$ di $\overline{a b}$.

(1) Annato Denjox, Mémoire sur les nombres dérivés des fonctions continues, in "Journal de Mathématiques pures et appliquées ", annee 1915, tome I, pag. 149.

(?) "Derivato mediano destro" è uno qualunque dei valori limiti del rapporto incrementale destro, che non sia nè $D_{+}$nè $D_{+}$(DExJor, loc. cit., pag. 145).

$\left(^{3}\right)$ Secondo il DexJoY (loc. cit., pag. 123) si chiama «residuale» di un aggregato perfetto $\mathfrak{F}$ (continuo o no) il subaggregato di $\mathscr{P}$ che rimane quando si escludano da $\mathfrak{F}$ succes. 
Drm. Sia $\varepsilon_{\imath}, \varepsilon_{2}, \ldots, \varepsilon_{n}, \ldots$ una successione di numeri positivi tendente a 0 . Avendo posto

$$
l_{n}=\frac{F\left(d_{n}\right)-F\left(c_{n}\right)}{d_{n}-c_{n}}
$$

considero il rapporto incrementale $\frac{F\left(d_{n}\right)-F(x)}{d_{n}-x}$ con $x$ variabile nelle vicinanze di $c_{n}$ per valori maggiori di $c_{n}$. Poichè, per ipotesi, $F(x)$ è continua verso destra in $c_{n}$, esiste un intorno destro di $c_{n}$ (segmento avente $c_{n}$ per estremo sinistro) che indico con $\sigma_{n} \equiv \overline{c_{n} c_{n}^{\prime}}$, minore di $\varepsilon_{n}$, avente per estremo destro $c_{n}^{\prime}$ compreso fra $c_{n}$ e $d_{n}$, e tale che, per ogni suo punto $x$, il quoziente considerato è compreso fra $l_{n}-\varepsilon_{n}$ ed $l_{n}+\varepsilon_{n}$.

Dico che ogni punto $\xi$ appartenente a un'infinita di $\sigma_{n}$ appartiene ad $E$. Infatti, anzitutto, se $\sigma_{n}$ contiene $\xi$, l'estremita destra $d_{n}$ dell'intervallo $S_{n}$ avente lo stesso indice di $\sigma_{n}$, supera $\xi$ e ne dista per meno di $S_{n}$. Dunque, per la successione delle $\sigma_{n}$ contenente $\xi, d_{n}$ tende a $\xi$ dalla destra. In secondo luogo il rapporto incrementale $\frac{F\left(d_{n}\right)-F(\xi)}{d_{n}-\xi}$ è, per la stessa successione di valori di $n$, compreso fra $l_{n}-\varepsilon_{n}$ ed $l_{n}+\varepsilon_{n}$. Dunque tutti i suoi valori limiti che sono dei derivati destri mediani od estremi di $F(x)$ in $\xi$, sono compresi nell'insieme dei valori limiti della successione $l_{n n}$. Se dunque a) $l_{n}$ supera $k$, $F(x)$ possiede in $\xi$ un derivato mediano o estremo almeno uguale a $h \mathrm{e}$ perciò si ha $\bar{D}_{+} F(\xi) \geq k$; se b) $l_{n}<k, \underline{D}_{+} F(\xi) \leq k$, e se $\left.c\right) \lim _{n \rightarrow \infty} l_{n}=l$ finito od infinito, $\underline{D}_{+} F(\xi) \leq l \leq \bar{D}_{+} F(\xi)$, e precisamente $l$ è un derivato mediano o estremo destro.

L'aggregato dei punti interni a un'infinità d'intervalli $\sigma_{n}$ è ovunque denso su $\overline{a b}$. Infatti: sia $\overline{m n}$ un qualunque segmento parziale di $\overline{a b}$. Sia $c_{n_{1}}$ il primo punto della successione $c_{1}, c_{2}, \ldots, c_{n}, \ldots$ interno ad $\overline{m n}$ e tale che $\sigma_{n_{1}}$ sia interamente contenuto in $\overline{m n}$. Sia $c_{n_{2}}$ il primo punto della medesima successione $c_{1}, c_{2}, \ldots, c_{n}, \ldots$ interno a $\sigma_{n_{1}}$ e tale che $\sigma_{n_{2}}$ sia interamente contenuto in $\sigma_{n_{1}}$. Cosi si prosegue costruendo una successione d'infiniti $\sigma_{n_{4}}, \sigma_{n_{3}}, \sigma_{n_{3}}, \ldots$

sivamente un' infinità numerabile di aggregati non densi in $\mathscr{B}$. Seguendo R. BAIRE (Sur les fonctions de variables réelles, in "Annali di Matematica ", serie IIT, tomo III (1899), pag. 67) si può dire che un "residuale * di un aggregato perfetto $\mathscr{P}$ è il subaggregato di $\mathscr{P}$ che è complementare di un subaggregato di prima categoria su $\mathfrak{B}$. Si dimostra (DenJor, loc. cit., pag. 232; BAIRE, loc. cit., pag. 67) che "ogni residuale d' un insieme perfetto con. tiene esso stesso un insieme perfetto $\%$ 
ciascuno contenuto nel precedente e in $\overline{m n}$. Questa successione ha per limite un punto $\xi$ di $E\left({ }^{4}\right)$. Dunque $E$ ha punti entro $\overline{m n}$ e perció, per l'arbitrarietà della scelta di $\overline{m n}, E$ è ovunque denso in $\overline{a b}$.

Resta da dimostrare che $E$ è un residuale di $\overline{a b}$. Infatti, essendo le $\sigma_{n}$ definite come sopra, consideriamo l'insieme $H_{0}$ dei punti di $\overline{a b}$ che non sono interni a nessuno dei segmenti $\sigma_{1}, \sigma_{2}, \ldots, \sigma_{n}, \ldots$ Questo insieme è evidentemente chiuso perchè un punto estraneo ad $H_{0}$, essendo interno a un intervallo $\sigma_{n}$ interamente estraneo ad $H_{0}$, non può essere punto limite di $H_{0}$. Dico che $H_{0} \dot{e}$ non denso in $a \dot{b}$. In caso contrario esisterebbe un segmento $\overline{m n}$ appartenente interamente ad $H_{0}$. Ora l'insieme $c_{n}$ essendo denso in $\overline{a b}$ esiste in $\overline{m n}$ almeno un punto $c_{n}$. L'intervallo $\sigma_{n}$ corrispondente, avendo per estremo $c_{n}$, contiene tutta una porzione di $\overline{m n}$. Dunque $H_{0}$ è non denso in $\overline{a b}$. Se noi sopprimiamo un numero finito di punti $c_{n}$, questo insieme non cessa di essere denso. Sia dunque $H_{p}$ l'insieme dei punti di $\overline{a b}$ che non sono interni a nessuno dei segmenti $\sigma_{p+1}, \sigma_{p+2}, \ldots H_{p}$, che contiene $H_{p-1}$, è non denso in $\overline{a b}$ (e d'altra parte chiuso). Consideriamo l'insieme $H$ costituito dalla riunione degli aggregati $H_{0}, H_{1}, H_{2}, \ldots, H_{p}, \ldots$ Sia $G$ il residuale di $\overline{a b} \mathrm{com}$ plementare di $H$. Ogni punto $M$ di $G$ è interno a un'infinità d'intervalli $\sigma_{n}$. Poichè se questo punto $M$ fosse interno soltanto a un numero finito di essi, il più grande degl' indici di questi avrebbe un certo valore $p . M$ apparterrebbe a tutti gl'insiemi $H_{p+1}, H_{p+2}, \ldots$, dunque ad $H$ e non a $G$. Dunque $M$ appartiene all'insieme $E$ qualificato nell' enunciato. Dunque $E$ contiene $G$. Inversamente si vede subito che $G$ contiene $E$. Dunque $G \equiv E$, ed $E$ è un residuale di $\overline{a b}$.

c. d. d.

Seguendo le tracce del Denjor possiamo dedurre dal teorema fondamentale delle importanti applicazioni. Ma non ne riportiamo tutte hè interamente le dimostrazioni per le quali rimandiamo alla citata memoria del Densor. Ci soffermeremo invece su quelle osservazioni che maggiormente interessano il nostro studio.

3. (Denjoy, loc. cit., pag. 152). Supponiamo verificata l'ipotesi c) del teorema fondamentale relativamente a due sistemi d'intervalli $S_{n 2}$ ed $S_{12}$. Supponiamo cioè che il rapporto incrementale $l_{n}$ relativo ad $S_{n}$ tenda ad un

(4) Che il punto $\xi$ appartenga ad $E$ si vede facilmente. Infatti gli estremi $c_{n 1}, c_{\eta 2}, c_{n 3}, \ldots$ costituiscono una successione crescente, gli estremi $d_{\eta_{1}}, d_{\eta 2}, d_{n 2}, \ldots$ una successione decrescente; e queste due successioni sono nelle condizioni d'applicabilità del postulato di DEDEKIND : esse individuano un punto limite $\xi$ che è realmente interno a infiniti $\sigma_{k}$. 
limite unico finito od infinito $l$, che il rapporto incrementale $l_{n}$ relativo ad $S_{n}^{\prime}$ tenda ad un limite unico finito od infinito $l^{\prime} \neq l$. Tndichiamo gli elementi omologhi relativi al sistema $S_{n}{ }^{\prime}$ con gli stessi simboli adoperati per gli elementi relativi al sistema $S_{n}$, distinguendoli mediante un accento: cosi gli elementi $\sigma_{n k}{ }^{\prime}, c_{n}{ }^{\prime}, d_{n}{ }^{\prime}$. Riprendiamo la dimostrazione del teor. fondamentale applicandola alternativamente alla successione $c_{n}$ e alla successione $c_{n z}^{\prime}$. Veniamo allora a determinare una successione di segmenti $\overline{m n}, \sigma_{n_{1}}, \sigma_{n_{1}}^{\prime}, \sigma_{n_{4}}$, $\sigma_{n_{3}}^{\prime}, \sigma_{n_{3}}, \sigma_{n_{3}}^{\prime}, \ldots$ ciascuno contenuto nel precedente. I segmenti $\sigma_{n_{1}}^{\prime}, \sigma_{n_{3}}^{\prime}, \sigma_{n_{3}}^{\prime}, \ldots$ sono scelti fra $i \sigma_{n}{ }^{\prime}$ cosi come i segmenti $\sigma_{n_{3}}, \sigma_{n_{2}}, \sigma_{n_{3}}, .$. , sono scelti fra i $\sigma_{n}$. La possibilità di determinare questa successione risulta dalla presenza, su ogni intervallo parziale di $a b$, degl'insiemi $c_{n}$ e $c_{n}^{\prime}$ separatamente. La successione $\sigma_{n_{1}}, \sigma_{n_{1}}^{\prime}, \sigma_{n_{2}}, \sigma_{n_{2}}^{\prime}, \ldots$ tende ad un punto. limite $\xi$ appartenente ad $\overline{a b}$, interno a un'infinità di $\sigma_{i n}$, a un'infinità di $\sigma_{i z}^{\prime}$, e dove, quindi, $F$ ammette per numeri derivati mediani o estremi destri sia $l$ che $l^{\prime}$.

Si deduce il corollario seguente:

(DENJor, loc. cit., pag. 156). Se una funzione ammette da una stessa parte una derivata unica in ogni punto di $\bar{a} \bar{b}$, è impossibile che i due aggregati in cui questa derivata è $l$ per il primo, $l^{\prime}$ per il secondo, siano l'uno e l'altro densi su $\bar{a} b$.

4. (Denjoy, loc. cit., pag. 15̃3). Se $l_{4 x}$ è un numero funzione dell' intervallo $S_{n} \equiv{\overline{c_{n} d_{n}}}_{n}$, la cui lunghezza tende a 0 per $n$ tendente ad $\infty$ ( $l_{n}$ essendo più precisamente in questo studio il rapporto incrementale di $F(x)$ su $S_{n}$, , conveniamo di dire che "l'insieme $l_{n}$ ammette in un punto $M$ di $\overline{a b}$ il valor limite $\lambda \otimes$ finito od infinito, se esiste una successione scelta fra i $c_{n}$ e tendente ad $M$ iu modo che i numeri $l_{n}$ tendano a $\lambda$.

I punti di $\overline{a b}$ analoghi ad $M$, in cui l'insieme $l_{n}$ ammette il valor limite $\lambda$, formano evidentemente un insieme chiuso. Supponiamo quest' ultimo coincidente con $\overline{a b}$. Qualunque sia $\varepsilon$, é possibile trovare, vicino quanto si vuole ad un punto $M$ qualunque di $\overline{a b}$, un punto $c_{n}$ tale che, per lo stesso indice $n, l_{n}$ differisca da $\lambda$ per meno di $\varepsilon$, se $\lambda$ è finito (sia del segno di $\lambda$ e sorpassi $\frac{1}{\varepsilon}$ in valore assoluto, se $\lambda$ è infinito). Scegliamo su $\overline{a b}$ una successione $\varphi_{1}, \varphi_{2}, \ldots$ ovunque densa su $\overline{a b}$ e a ciascun $\varphi_{12}$ facciamo corrispondere un numero positivo qualunque $\eta_{n z}\left(\lim _{n \rightarrow \infty} \eta_{n i}=0\right)$. Estragghiamo poi dalla successione $c_{1}, c_{2}, \ldots, c_{i n}, \ldots$ una succersione $\gamma_{1}, \gamma_{2}, \ldots, \gamma_{n}, \ldots$ in modo che, per 
ogui $n$, sia

$$
\left|\varphi_{n}-\gamma_{n}\right|<\eta_{n} \text { e }\left|\lambda-\lambda_{n}\right|<\eta_{n}
$$

( $\left|\lambda_{n}\right|>n$ e $\lambda_{n}$ del segno di $\lambda$, se $\lambda$ è infinito), avendo indicato con $\lambda_{n}$ il valore di $l_{n}$ che corrisponde a $\gamma_{n}$. E chiaro che i punti $\gamma_{n}$ sono densi su $\overline{a b}$ e che $\lambda_{n}$ tende a $\lambda$. Il teor. fondamentale ci permette immediatamente di concludere:

Se $S_{n}$ è una successione d'intervalli aventi lunghezze tendenti a 0 quando $n$ tende ad $\infty$, e il cui estremo sinistro descrive un insieme ovunque denso in $\overline{a b}$, se il rapporto incrementale $l_{n}$ di $F(x)$ su $S_{n}$ ammette in ogni punto di $\overline{a b}$ il valor limite $\lambda$, finito od infinito, sarà $\lambda$ un derivato mediano o estremo destro di $F(x)$ in ogni punto di un residuale di $\overline{a b}$.

Quando è $\lambda=+\infty(-\infty)$, diciamo che $l_{y z}$ è non limitato superiormente (inferiormente) in ogni porzione di $\overline{a b}$ od in vicinanza di ogni punto di $\overline{a b}$. In questo caso $F(x)$ ammette la derivata superiore (inferiore) destra $+\infty(-\infty)$ in un residuale di $\overline{a b}$.

5. (DENJoY, loc. cit., pag. 154). Se l'insieme $E(l)$ dei punti in eui $F(x)$ ha per derivato mediano o estremo destro un numero $l$ è ovunque denso su $\overline{a b}$, questo insieme è un residuale di $\overline{a b}$.

Infatti essendo $E(l)$ ovunque denso in $\overline{a b}$, noi possiamo estrarre da esso un'infinita numerabile di punti $c_{1}, c_{2}, \ldots, c_{n}, \ldots$ ovunque densa in $\overline{a b}\left(5^{5}\right)$. $l$ essendo in $c_{n}$ un derivato destro, esiste un intervallo $\overline{c_{n} d_{n}}$ di estremo sinistro $c_{n}$, di lunghezza inferiore ad $\varepsilon_{n}\left(\varepsilon_{i}, \varepsilon_{2}, \ldots, \varepsilon_{n}, \ldots\right.$ successione di numeri positivi tendente a $O$, prefissata ad arbitrio), in cui il rapporto incrementale di $F(x)$ differisce da $l$ per meno di $\varepsilon_{n z}$, se $l$ è finito, è del segno di $l$ e superiore in valore assoluto ad $n$, se $l$ è infinito. Siamo allora nelle condizioni d'applicabilità del teorema fondamentale: l'insieme $E(l)$ è dunque dappertutto un residuale.

Sono dunque dei residuali di $\overline{a b}$, tosto che essi siano ovunque densi in $\overline{a b}$, gli insiemi in cui 0 è un derivato mediano destro, $0 F(x)$ ha il suo derivato superiore destro $+\infty$, il suo derivato inferiore destro - $\infty$.

(5) Infatti dividiamo $\overline{a b}$ in $n$ parti nguali e su ciaseuna di esse scegliamo indifferen. temente un punto di $E(l)$. L'insieme dei punti scelti per tutti i valori di $n$ è evidentemente numerabile, ovunque denso in $\bar{a} \bar{b}$. La dimostrazione fa manifestamente uso del postrulato di ZerMelo, ma questo uso è legittimo, perchè esso conduce non ad una "costruzione " bensì ad una "dimostrnzione ", la quale risulta vera indipendentemente dalle scelte eseguite. 
6. (Denjoy, loc. cit., pag. 156). Se $D_{+} F(x)$ è finita in ogni punto di $\overline{a b}$, l' insieme dei punti di $\overline{a b}$ in vicinanza dei quali $\bar{D}_{+} F(x)$ è non limitata superiormente è non denso in $\overline{a b}$.

7. Confrontando il teorema fondamentale da noi dimostrato al $11 .^{\circ} 2$ con l'originale del DenJoy osserviamo che, quanto alla generalità, esso pressappoco gli equivale. Infatti gli toglie la condizione restrittiva della continuita verso sinistra, ma glie ne sostituisce un'altra, quella che l'aggregato perfetto $\mathfrak{B}$, a cui deve appartenere la successione $c_{1}, c_{2}, \ldots, c_{n}, \ldots$ e in cui la medesima successione deve essere densa, sia l'intero segmento $\bar{a} \vec{b}$. Questa condizione puó sostituirsi con altra equivalente, ove l'aggregato perfetto $\mathfrak{B}$ si supponga discontinuo. Osserviamo infatti che, per la validità del ragionamento del $1 .^{\circ} 2$, occorre che di ogni $c_{n}$ esista un intorno $\sigma_{n}$ sufficientemente piccolo soddisfacente a due condizioni essenziali: $\left.1^{\mathrm{a}}\right) \sigma_{n}$ contiene altri infiniti punti $\left.c_{n} ; 2^{a}\right) F(x)$ varia in $\sigma_{n}$ di quanto poco si vuole.

Se dunque si suppone, come al $n .^{\circ} 2$, che $\mathfrak{B}$ sia continuo, queste due condizioni sono soddisfatte per un intorno destro $\sigma_{n}$ di $c_{n}$. Se si suppone che $\mathfrak{B}$ sia discontinuo, occorre accertarsi di quelle condizioni, perchè esse non sono soddisfatte senz' altro.

Così ad es., supposto $\mathfrak{B}$ perfetto e ovunque non denso in $\bar{a} b$, se la successione $c_{1}, c_{2}, \ldots, c_{n}, \ldots$ nelle condizioni del teorema relativamente a $\mathfrak{B}$, non contiene nessun punto che sia estremo sinistro di un intervallo contiguo a $\mathfrak{B}$ (o al piu ne contiene un numero finito, od anche infiniti, ma in condizioni che si tratterebbe di precisare) $\left({ }^{6}\right)$, allora il teorema fondamentale ville ugualmente relativamente a $\mathfrak{B}\left({ }^{7}\right)$, e valgono quindi tutte le conclusioni che ne abbiamo tratte.

Se in tutti i punti di $\mathscr{P}$ che siano estremi sinistri d'intervalli contigui, la $F(x)$ è continua verso sinistra, il teorema fondamentale vale anche quando la successione $c_{1}, c_{2}, \ldots, c_{n}, \ldots$ contenga degli estremi sinistri d'intervalli contigui a $\mathfrak{B}$ (infiniti di questi estremi, ovunque densi in $\mathfrak{S}$ ). Basta infatti, qualora, ripetendo i ragionamenti del $n .^{\circ} 2$, ci s'imbatta nella scelta di qualche $c_{n 2}$ che sia estremo sinistro di un intervallo contiguo a $\mathfrak{B}$, assumere per intervallo $\sigma_{n}$ un conveniente intorno sinistro di $c_{n}$.

(6) $\mathrm{E}$ noto che un aggregato perfetto e uon denso è costituito da tutti e soli i punti che non sono interni a un sistema d'intervalli (detti " contigui all'aggregato perfetto ") ovunque densi sul continuo.

(7) La tesi afferma allora l'esistenza dell' aggregato $E$ denso in $\mathfrak{B}$, ed anzi residuale di $\mathfrak{B}$ 


\section{§ 2. Aggregato dei punti di discontinuità. Esempî.}

1. Definizione. - - Se $\varphi(x)$ è una funzione definita in un intervallo $\overline{m n}$, seguendo la nomenclatura di uso corrente chiameremo * oscillazione di $\varphi(x)$ in $\overline{m n}$, la differenza fra il limite superiore di $\varphi(x)$ e il limite inferiore di $\varphi(x)$ in $\overline{m n}$. Se gli estremi $m, n$ dell' intervallo $\overline{m n}$ si suppongono variabili e precisamente tendenti ad un determinato punto $x_{0}$ interno all'intervallo stesso (cioè in modo che $m$ tenda a $x_{0}$ dalla sinistra ed $n$ dalla destra) allora l'oscillazione di $\varphi(x)$ in $\overline{m n}$ tende ad un valore limite ben determinato (ed indipendente dal modo di variare di $m, n$ ) che chiameremo "oscillazione (puntuale) di $\varphi(x)$ in $x_{0}$ s.

2. Se $\varphi(x)$ è una funzione derivabile verso destra nel punto $x_{0}$ e se $\omega(x)$ è la sua oscillazione puntuale (considerata come funzione della $x$ ), è

$$
\lim _{x \rightarrow x_{0}+0} \frac{\omega(x)}{x-x_{0}}=0
$$

Infatti è, per definizione della derivata destra, $\left(h_{1}>0, h_{2}>0\right)$

$$
\begin{aligned}
& \varphi\left(x+h_{1}\right)=\varphi\left(x_{0}\right)+\left(x+h_{1}-x_{0}\right) D_{+} \varphi\left(x_{0}\right)+\left(x+h_{1}-x_{0}\right) \varepsilon_{1}, \\
& \varphi\left(x+h_{2}\right)=\varphi\left(x_{0}\right)+\left(x+h_{2}-x_{0}\right) D_{+} \varphi\left(x_{0}\right)+\left(x+h_{2}-x_{0}\right) \varepsilon_{2},
\end{aligned}
$$

$\varepsilon_{1}$ ed $\varepsilon_{2}$ infinitesimi rispettivamente con $\left(x+h_{1}-x_{0}\right),\left(x+h_{2}-x_{0}\right)$. Segue (2) $\varphi\left(x+h_{1}\right)-\varphi\left(x+h_{2}\right)=\left(h_{1}-h_{2}\right) D_{+} \varphi\left(x_{0}\right)+\left(x+h_{1}-x_{0}\right) \varepsilon_{1}-\left(x+h_{2}-x_{0}\right) \varepsilon_{2}$.

Perció, potendosi scrivere

$$
\omega(x)=\varlimsup_{\substack{h_{1} \rightarrow 0 \\ h_{2} \rightarrow 0}}\left|\varphi\left(x+h_{1}\right)-\varphi\left(x+h_{2}\right)\right|
$$

è $\omega(x)=\left(x-x_{0}\right) \varepsilon$, essendo $\varepsilon$ infinitesimo con $x-x_{0}$. Segue l'enunciato.

Osservazione. L'oscillazione puntuale $\omega(x)$ potrebbe definirsi « l'oseillazione di $\varphi(x)$ in un intorno nullo del punto $x$. La formola dimostrata vale anche nell'ipotesi meno stretta che $\omega(x)$ sia l'oscillazione di $\varphi(x)$ in un intomo non nullo del punto $x$, purchè sufficientemente piccolo: per es. di ampiezza inferiore ad $\left(x-x_{0}\right)^{2}$. Poniamo

$$
\underset{-\delta \leq\left(h_{1}, h_{2}\right) \leq \delta<\left(x-x_{0}\right)^{2}}{\omega} \varlimsup_{\left.-h_{1}\right)-\varphi\left(x+h_{2}\right) \mid .} \mid \varphi(x)
$$


Dalla (2) segue allora

$$
\frac{\omega(x)}{x-x_{0}} \leq 2\left(x-x_{0}\right) ; D_{+} \varphi\left(x_{0}\right) \mid+2\left(\left|\varepsilon_{1}\right|+\left|\varepsilon_{2}\right|\right)
$$

e quindi l'enunciato.

3. Ritorniamo ora ad una generica funzione $F(x)$, continua verso destra in tutto un intervallo $\overline{a b}$. Ci proponiamo di studiare la struttura dell'aggregato dei suoi punti di discontinuita.

I punti di discontinuitá della $F(x)$ sono soltanto punti di discontinuita verso sinistra. Essi si possono distinguere in punti di discontinuità di $1^{a}$ specie (o « salti $*$, per i quali esiste il

$$
\lim _{h \rightarrow-10} F(x-h)=E(x-0),
$$

e in punti di discontinuità di $2^{a}$ specie, per i quali tale limite non esiste.

Indichiamo con $\mathscr{T}$ l'aggregato dei punti di discontinuita di $F(x)$ in $\overline{a b}$.

4. Teor. L'aggregato or dei punti di discontinuitá di $F(x)$ in $\overline{\imath b}$ è numerabile.

DrM. Assegnato ad arbitrio un numero $\eta>0$, ad ogni punto di $\overline{a b}$ si può far corrispondere un intorno destro (segmento avente quel punto per estremo sinistro) in cui l'oscillazione di $F(x)$ è $<\eta$. I punti in cui l'oscillazione a sinistra $\dot{e}>\eta \eta$ sono tutti estremi sinistri di tali segmenti. Formiamo l'aggregato dei segmenti che si ottengono riunendo in un solo tutti quelli che hanno punti comuni (interni od estremi). I punti in cui l'oscillazione a sinistra $\dot{e}>\eta$ risultano allora estremi di un sistema di segmenti senza punti comuni e perció costituiscono un aggregato numerabile $\left({ }^{8}\right)$.

Ripetendo il ragionamento successivamente per

$$
\frac{\eta}{2}, \frac{\eta}{4}, \ldots, \frac{\eta}{2^{n}}, \ldots
$$

si vengono a classificare tutti $\mathrm{i}$ punti di $\mathscr{\mathscr { C }}$ in una successione di aggregati numerabili, il primo dei quali è costituito dai punti di $\mathscr{C}$ in cui l'oscillazione a sinistra è maggiore di $\eta$, il secondo dai punti di $\mathscr{\mathscr { C }}$ in cui l'oscillazione a sinistra è compresa fra $\eta$ ed $\frac{\eta}{2}$, il terzo dai punti di $\mathscr{O}$ in cui l'oscillazione

$\left.{ }^{8}\right)$ Per un noto teor. di Cantor: un insieme d'intervalli di una retta, non sovrapponentisi, è numerabile. 
a sinistra è compresa fra $\frac{\eta}{2}$ ed $\frac{\eta}{4}, \ldots, \mathrm{l}^{\prime}(n+1)^{m o}$ dai punti di $\mathcal{O}$ in cui l'oscillazione a sinistra è compresa fra $\frac{\eta}{2^{n-1}}$ ed $\frac{\eta}{2^{n}}$. Dunque or è numerabile.

c. d. d.

5. Studiamo anzitutto il caso che $\mathscr{Q}$ sia denso in qualche parte $\overline{m n}$ di $\overline{a b}$. In tale ipotesi, supponiamo $\mathscr{O}$ numerato (com'è sempre possibile per il teor. del n. prec.) nella successione $d_{1}, d_{2}, \ldots, d_{n}, \ldots$ Sin $\varepsilon_{1}, \varepsilon_{2}, \ldots, \varepsilon_{n}, \ldots$ una successione di numeri positivi tendente a $O$. A sinistra di $d_{1}$ esiste celtamente un punto $c_{1}$ tale che $d_{1}-c_{1}<\varepsilon_{1}$ e che il rapporto incrementale $l_{1}$ di $F(x)$ su $\overline{c_{1} d_{1}}$ sia, in valore assoluto, $>\frac{1}{\varepsilon_{1}}$. A sinistra di $d_{2}$ esiste certamente un punto $c_{2}$ tale che $d_{2}-c_{2}<\varepsilon_{2}$ e che il rapporto incrementale $l_{2}$ di $F(x)$ su $\overline{c_{2} d_{2}}$ sia, in valore assoluto, $>\frac{1}{\varepsilon_{2}} \ldots$. In generale, a sinistra di $d_{n}$ esiste certamente un punto $c_{n}$ tale che $d_{n}-c_{n}<\varepsilon_{n}$ e che il rapporto incrementale $l_{n}$ di $F(x)$ su $\overline{c_{n} d_{s b}}$ sia, in valore assoluto, $>\frac{1}{\varepsilon_{n}}$.

I segmenti $S_{n} \equiv \overline{c_{n} l_{n}}$, per $n=1,2,3, \ldots$ sono nelle condizioni del teor. fondamentale $\left(\$ 1\right.$, n. $^{\prime \prime}$ 2) $\left({ }^{9}\right)$. Si conclude dunque col seg.

Teor. Se $\mathscr{T}$ è deuso in un segmento $\overline{m n}$ parziale di $\overline{a b}$ (eventualmente in tutto $\overline{a b}$ ) l'aggregato dei punti $x$ in cui è verificata almeno una delle due uguaglianze

$$
\bar{D}_{+} F(x)=+\infty, \quad D_{+} F(x)=-\infty
$$

è denso in $\overline{m n}$ ed anzi è un residuale di $\overline{m n}\left({ }^{10}\right)$.

Con. Se i numeri derivati destri di $F(x)$ sono ovunque finiti in $\bar{a} \vec{b}, l^{\prime}$ aggregato $\mathscr{T}$ dei punti di discontinuità di $F(x)$ è ovunque non denso in $\overline{a b}$.

$\left({ }^{9}\right)$ Si può sempre fare in modo che i punti $c_{n}$ appartengano all'intervallo $\overline{m n}$ (evidentemente questa condizione non è neanche necessaria, poichè basterebbe, in caso contrario, trascurare tutí i $c_{n}$ che stanno fuori di $\overline{n n}$ ). Essi costituiseono una suecessione densa in $\overline{m n}$. Infatti se $\bar{r} s$ è un intervallo parziale di $\overline{m n}$ preso ad arbitrio, esistono nel terzo medio di $\overrightarrow{r s}$ infiniti punti $d_{n}$ : di questi, tutti quelli che hanno indice $n$ sufficientemente elevato perehè $\varepsilon_{n}$ sia inferiore ad $\frac{s-r}{3}$ hanno per corrispondenti dei punti $c_{n}$ che sono interni ad $\overline{r s}$.

(10) Vedi la nota alla fine del n. ${ }^{\circ} 11$. 
6. Indichiamo con $\mathcal{O K}_{\ddagger}$ l'aggregato dei punti di discontinuita di $1^{*}$ specie per la $F(x)$ in $\overline{a b}$, con $\mathscr{O}$, quello dei punti di discontinuita di $2^{n}$ specie.

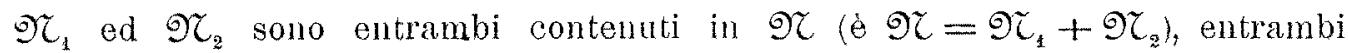
numerabili.

Sia $\mathscr{\mathscr { C }}_{z}$ denso in qualche parte $\overline{m n}$ di $\overline{a b}$, numerato nella successione $h_{1}, h_{2}, \ldots, h_{n}, \ldots$. Sia $\tau_{1}, \tau_{2}, \ldots, \tau_{n}, \ldots$ una successione di numeri positivi tendente a 0 . Nell'intervallo comune ai due intervalli $h_{1}-\overline{\tau_{1} h_{1}}, \overline{m n}$, esiste una coppia (anzi esistono infinite coppie) d'intervalli $S_{1}^{*}, S_{1}^{\prime}$ tali che i rispettivi rapporti incrementali $l_{1}, l_{1}^{\prime}$ di $F(x)$ siano il primo maggiore di +1 , il secondo minore di $-1\left({ }^{11}\right)$. Nell' intervallo comune ai due intervalli $h_{2}-\overline{\tau_{2} h_{2}}$, $\overline{m n}$, esiste una coppia d'intervalli $S_{2}, S_{2}^{\prime}$ tali che i rispettivi rapporti incrementali $l_{2}, l_{2}^{\prime}$ di $F(x)$ siano il primo maggiore di +2 , il secondo minore di - 2. Cosi si prosegua: in generale nell' intervallo comune ai due intervalli $h_{n}-\overline{\tau_{n} h_{n}}, \overline{m n}$, esiste una coppia d'intervalli $S_{n}, S_{n}{ }^{\prime}$ tali che i rispettivii rapporti incrementali $l_{n}, l_{n}^{\prime}$ di $F(x)$ siano il primo maggiore di $+n$, il secondo minore di $-n$.

I due sistemi d'intervalli $S_{1}, S_{2}, \ldots, S_{32}, \ldots, S_{1}{ }^{\prime}, S_{2}{ }^{\prime}, \ldots, S_{n}{ }^{\prime}, \ldots$, ovunque densi in $\overline{m n}\left({ }^{12}\right)$, sono nelle condizioni d'applicabilità del teor. fondamentale e precisamente delle considerazioni del $\S 1, n .^{\circ} 3$. Si deduce quindi il

Teor. Se l'aggregato $\mathscr{Q}_{z}$ dei punti in cui $F(x)$ ha una discontinuità di $2^{a}$ specie é denso in un segmento $\overline{m n}$ parziale di $\overline{a b}$ (eventualmente in tutto $\overline{a b})$, l'aggregato dei punti $x$ in cui sono verificate entrambe le uguaglianze

$$
\bar{D}_{+} F(x)=+\infty, \quad \underline{D}_{+} F(x)=-\infty,
$$

è denso in $\overline{m n}$, ed anzi è un residuale di $\overline{m n}$.

(11) Infatti sia

$$
\bar{\lambda}=\varlimsup_{x \rightarrow h_{1}-0} F(x), \quad \lambda=\lim _{x \rightarrow h_{1}-0} F(x),
$$

e supponiamo, per fissare le idee, che tanto $\bar{\lambda}$ e $\lambda$ siano finiti. Poniamo $\varepsilon=\frac{\bar{\lambda}-\lambda}{3}$. Sia $k$ il massimo dei tre numeri

$$
h_{1}-\tau_{1}, m, h_{1}-\varepsilon .
$$

Siano $\alpha_{1}<\alpha_{2}<x_{3}<\alpha_{1}$ quatiro punti interai all intervallo $\bar{k} h_{1}$ e tali che

$$
\left|\underline{\lambda}-F\left(x_{1}\right)\right|<z ; \quad\left|\bar{\lambda}-F\left(x_{2}\right)\right|<\varepsilon, \quad\left|\bar{\lambda}-F\left(x_{3}\right)\right|<\varepsilon, \quad \mid \underline{\lambda}-F\left(x_{4}\right)<\varepsilon .
$$

Il rapporto incrementale di $F(x)$ è $>1$ nell intervallo $\overline{x_{1} x_{2}}$, è $<-1$ nell intervallo $\overline{x_{3} x_{4}}$.

La dimostrazione si estende in modo orvio al caso che uno od ambedue i numeri $\bar{\lambda}, \lambda$ siano infiniti.

("*) Vedi la nota (9). 
Cok. Se in ogni punto di $\overline{a b}$ almeno uno dei numeri derivati estremi destri di $F(x)$ è finito, l'aggregato $\mathscr{T}_{2}$ dei punti di discontinuità di $2^{\text {a }}$ specie di $F(x)$ è ovunque non denso in $\overline{a b}$.

Cor. Se $F(x)$, oltrecchè continua, è anche ovunque derivabile verso destra in $\overline{a b}$, allora l'aggregato $\mathscr{T C}_{2}$ dei punti di discontinuita di $2^{\mathrm{a}}$ specie di $F(x)$ è ovunque non denso in $\overline{a b}$.

Si puó nella stessa ipotesi affermare la stessa tesi per l'aggregato $\mathfrak{O} \mathcal{C}_{1}$ dei punti di discontinuita di $1^{2}$ specie? Non certo in base alle considerazioni dei $n .^{i} 5$ e seg., ma in base alla formola del $11 .{ }^{\circ} 2$, come ora precisamente vogliamo mostrare.

7. TeOr. Se $F(x)$ è ovunque derivabile verso destra in $\overline{a b}$, l' aggregato $\mathscr{\Upsilon}$ dei punti di discontinuità di $F(x)$ è ovunque non deuso su $\overline{a b}\left({ }^{13}\right)$.

Dim. Ragioniamo per assurdo. Supponiamo, se possibile, che $\mathscr{O}$ sia denso su una parte $\overline{m n}$ di $\overline{a b}$. Siano

$$
\begin{array}{ll}
\omega_{1}, & \omega_{2}, \ldots, \omega_{n}, \ldots \\
h_{1}, & h_{2}, \ldots, h_{n}, \ldots
\end{array}
$$

due successioni di numeri positivi tendenti a 0 , la seconda più rapidamente della prima. $\dot{\mathrm{E}}$ dunque, per ipotesi, $\lim _{n \rightarrow \infty} \frac{\omega_{n}}{h_{n}}=\infty$.

Indichiamo con o(x) l'oscillazione puntuale di $F(x)$ (considerata come funzione della $x$ ). Sia $a_{1}$ un punto qualsiasi di discontinuità per la $F(x)$, interno ad $\overline{m n}$ (per es. il primo che s'incontra nell'ordinamento di $\mathscr{Q C}$ ). Sia $r_{1}$ un indice (ad es, il più piccolo) tale che $\omega\left(a_{1}\right) \geq \omega_{r_{1}}$. Sia $a_{2}$ un punto qualsiasi di-discontinuitá per la $F(x)$, interno al segmento $\overline{m a}_{1}$ e tale che sia $a_{1}-a_{2}<h_{r_{3}}$. Sia $r_{2}$ un indice tale che $\omega\left(a_{2}\right) \geq \omega_{r_{3}}$. Sia $a_{3}$ un punto qualsiasi di discontinuità per la $F(x)$, interno al segmento $\overline{m a_{2}}$ e tale che sia $a_{1}-a_{3}<h_{r_{1}}, a_{2}-a_{3}<h_{r_{3}}, \ldots$. Cosi si prosegua: essendosi in generale scelto un $a_{n}$, si sceglierà un indice $r_{n}$ tale che $\omega\left(a_{n}\right) \geq \omega_{r_{n}}$, indi un $a_{n+1}$ interno al segmento $\overline{m a_{n}}$ e tale che sia $a_{1}-a_{n+1}<h_{r_{1}}, a_{2}-a_{n+1}<h_{r_{2}}, \ldots$, $a_{n}-a_{n+1}<h_{r_{32}}$.

I punti $a_{1}, a_{2}, \ldots, a_{n}, \ldots$ costituiscono una successione decrescente $\mathrm{e}$ quindi ammetteranno un punto limite $x_{0}(\geq m)$.

Posto $k_{n n}=a_{n}-x_{0}$, ̀̀ $k_{n} \leq h_{r_{n}}$ e quindi $\frac{\omega\left(a_{n}\right)}{k_{n}} \geq \frac{\omega_{r_{n}}}{h_{r_{n 2}}}$. Dunque, per l'ipo-

${ }^{\left({ }^{3}\right)}$ Questo teorema evidentemente racchiude il cor. $2^{\circ}$ del $n .^{0} 6$ come caso particolare. 
tesi fatta, è

$$
\lim _{n \rightarrow \infty} \frac{\omega\left(a_{n}\right)}{k_{n}}=\infty
$$

Ma questa conclusione è assurda perchè, contraddicendosi alla proposizione del $n^{\circ}{ }^{\circ} 2$, dovrebbe mancare la derivata destra di $F(x)$ nel punto $x_{0}$.

Osservazione. Nella dimostrazione non abbiamo contemplato il caso che in $x_{0}$ la derivata di $F(x)$ sia infinita: è evidente che allora la proposizione del 11. 2 non può applicarsi. In una Nota che pubblico nell' "Accademia dei Lincei sagiono in modo analogo dando la dimostrazione simultanea dei due teoremi qui dimostrati separatamente ai $n .^{i} 5,7$ e facendo osservare che nell' enunciato del presente numero può benissimo supporsi che in uno o più punti $x_{0}$ di $\overline{a b}$ la derivata destra di $F(x)$ sia infinita, purchè il rapporto incrementale tenda ad infinito « asintoticamente», cioè compiendo oscillazioni limitate.

Questa restrizione si dovrà perció supporre tacitamente nel seguito.

8. L'ipotesi assurda che sta alla base della dimostrazione del $n .^{\circ}$ precedente é anche troppo restrittiva. Può essere sostituita dall' ipotesi : ogni punto di $\mathscr{O}$ è limite verso la sua sinistra di punti di $\mathcal{O C}$; o dall'ipotesi: i punti di $\mathscr{K}$ che sono limiti verso la loro sinistra di punti di $\mathscr{O}$ costituiscono un aggregato ovunque denso in $\mathscr{\mathscr { C }}\left({ }^{14}\right)$.

Supponiamo $F(x)$ ovunque derivabile verso destra in $\overline{a b}$. Allora $\mathscr{G} \dot{\mathrm{e}}$ ovunque non denso in $\bar{a} \bar{b}$. Diciamo $\mathscr{V}^{(1)}$ l'aggregato dei punti ai $\mathscr{O C}$ che sono limiti verso sinistra di punti di $\mathcal{G}$. Ripetendo il ragionamento del $11 .{ }^{\circ} 7$ si vede che $\mathcal{O C}^{(1)}$ è ovunque non denso in $\mathscr{O}\left({ }^{(15}\right)$. Diciamo $\mathscr{O C}^{(2)}$ l'aggregato dei punti di $\mathscr{V}^{(1)}$ che sono limiti verso sinistra di punti di $\mathscr{\mathscr { C }}^{(1)}$. Anche $\mathscr{Q ⿻}^{(2)}$ è ovunque non denso in $\mathscr{T}^{(1)}$. Cosi si prosegua: in generale l'aggregato $\mathscr{T}^{(n)}$ dei punti di $\mathscr{T}^{(n-1)}$ che sono limiti verso sinistra di punti di $\mathscr{V}^{(n-1)}$ è ovunque non denso in $\mathscr{T}^{(n-1)}$.

Può darsi che esista un aggregato comune a tutti gli aggregati

$$
\mathscr{T}^{(1)}, \mathscr{T}^{(2)}, \ldots, \mathscr{O}^{(n-1)}, \mathscr{O}^{(n)}, \ldots
$$

In caso affermativo lo si indichi con $\mathscr{O}^{(\omega)}$. $\mathscr{O}^{(\omega)}$ sarà non denso in tutti

(44) Ció signifiea, com'è noto, che in ogni segmento $\bar{m} n$ contenente punti di $\mathfrak{O}$ esistono punti di $\mathscr{O}$ che sono limiti verso la loro sinistra di punti di $\mathscr{O}$.

(15) Ciò̀, entro ogni segmento $\overline{m n}$ contenente punti di $\mathscr{K}$, esiste un segmento parziale $\overline{r s}$, contenente punti di $\mathscr{Q}$, ma nessun punto di $\mathfrak{Q K}^{(1)}$. 
questi. Cosi si prosegua costruendo la successione transfinita di aggregati

$$
\mathscr{Y}^{(1)}, \mathscr{O}^{(2)}, \ldots, \mathscr{T}^{(\omega)}, \mathscr{T}^{(\omega+1)}, \ldots, \mathscr{O}^{(2 \omega)}, \ldots,
$$

ciascuno contenuto e non denso nei precedenti, mediante le due operazioni seguenti:

$1^{\text {a) }}$ Operazione $O_{1}$, che estrae da un aggregato $\mathscr{O}^{(n-1)}$ l'aggregato $\mathscr{O}^{(n)}$ dei punti limiti verso sinistra, per ogni $n$ intiero o transfinito di $1^{\text {a }}$ specie;

$\left.2^{a}\right)$ Operazione $O_{2}$, che estrae da una successione $d^{2}$ infiniti aggregati

$$
\mathscr{T}^{\left(r_{1}\right)}, \mathscr{O}^{\left(r_{2}\right)}, \ldots
$$

con indici $r_{1}, r_{2}, \ldots$ transfiniti crescenti, un aggregato $\mathscr{T}^{(\mu)}$ con indice transfinito di $2^{\mathrm{a}}$ specie.

Entrambe le operazioni $O_{1}, O_{2}$ possono avere per risultati aggregati nulli. Indichiamo con

$$
\operatorname{er}^{(1)}, \operatorname{err}^{(2)}, \ldots, \operatorname{egr}^{(\omega)}, \operatorname{egr}^{(\omega+1)}, \ldots, \operatorname{eV}^{(2 \omega)}, \ldots
$$

gli aggregati che si ottengono dai precedenti mediante chiusura ( $\left.{ }^{16}\right)$. La successione

$$
\mathfrak{e S}^{(1)}, \mathfrak{e V}^{(2)}, \ldots, \operatorname{eVT}^{(n-1)}, \mathfrak{C O T}^{(n)}, \ldots
$$

è una successione di aggregati chiusi, ciascuno contenuto $\left({ }^{17}\right)$ e non denso nel precedente. Sia $\mathcal{C}$ il suo aggregato limite $\left({ }^{18}\right)$. Evidentemente $\mathcal{C}$ contiene $\mathcal{C O C}^{(\omega)}$. Ma $\mathcal{C}$ è non denso in tutti gli aggregati

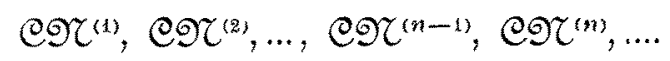

Dunque anche $\mathfrak{C O C}^{(\omega)}$ è non denso nei medesimi. Ne segue che la successione

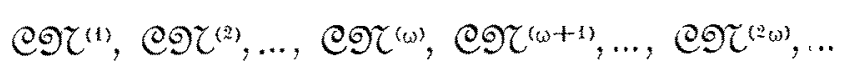

è «riduttibile» $\left({ }^{19}\right)$ e quindi è * riduttibile * anche la successione

$$
\mathscr{O}^{(1)}, \mathscr{O}^{(2)}, \ldots, \mathscr{O}^{(\omega)}, \mathscr{Y}^{(\omega+1)}, \ldots, \mathscr{N}^{(2 \omega)}, \ldots
$$

(16) Cioè con l'aggiunta dei punti limiti che eventualmente non appartengono a ciascuno di essi $\left(\mathcal{C O C}^{(n)}=\mathscr{V C}^{(n)}+\right.$ derivato di $\left.\mathscr{G}^{(n)}\right)$.

${ }^{(17)} \dot{\mathrm{E}}$ evidente che un punto limite di un aggregato $A$ contenuto in un aggregato $B$ è anche punto limite di $B$.

(18) È noto dalla teoria degli aggregati che una successione di aggregati chiusi, ciaseuno contenuto nel precedente, ammette sempre un aggregato limite $\mathfrak{C}$, cioè un aggregato chiuso contenuto in tutti gli aggregati della successione.

(17) Nella teoria degli aggregati il termine « riduttibile» si usa propriamente dire di un aggregato di cui un derivato è nullo. Qui lo usimo, per comodità, a proposito di una "successione "; nel senso che abbiamo indicato. 
La riduttibilità consiste nel fatto che esiste un transfinito ben determinato $\tau$ tale che $\mathcal{C Q}^{(\tau+1)} \dot{e}$ nullo ed $\mathcal{G}^{(\tau+1)}$ è nullo. Se $\tau$ è di prima specie $\dot{e}$ $\operatorname{COT}^{(\tau)} \neq 0$ ed anche $\mathscr{T}^{(\tau)} \neq 0$. Se $\tau \dot{e}$ di seconda specie può essere anche $\mathfrak{C O C}^{(\tau)}=\mathscr{T}^{(\tau)}=0$.

9. Le stesse osservazioni si possono fare a proposito del n. ${ }^{\circ} 5$; ma è necessario analizzare accuratamente la possibilità di ripetere la dimostrazione del n. ${ }^{\circ} 5$, perchè questa, come si è accennato alla fine del n. ${ }^{\circ} 6$ è (nonostante qualche analogia) di tutt'altra natura di quella del n. ${ }^{\circ} 7$ : quella del $n .^{\circ} 5$ è basata sul teorema fondamentale, mentre quella del n. 7 è basata sulla formola del $n .{ }^{\circ} 2$.

Supponiamo dunque che i numeri derivati estremi destri di $F(x)$ siano ovunque finiti in $\overline{a b}$. Allora $\mathscr{\mathcal { C }} \dot{\mathrm{e}}$ non denso in tutto $\overline{a b}$. Dico che $\mathscr{\Upsilon}^{(1)} \dot{\mathrm{e}}$ non denso in tutto $\mathscr{T}$. Supponiamo infatti, se possibile, che $\mathscr{T}^{(1)}$ sia denso sulla parte di $\mathscr{T}$ ch'è contenuta in un intervallo $\overline{n n}$ parziale di $\overline{a b}$ (eventualmente in tutto $\overline{a b}$ ). Sarà $\mathscr{G}^{(1)}$ denso anche nella parte dell'aggregato chiuso $\mathcal{C} \mathscr{C}$ che è contenuta in $\overline{m n}$. Inoltre la parte di $\mathcal{C O C}$ contenuta in $\overline{m n}$ sarà certamente perfetta.

Per ogni punto $\xi$ di $\mathscr{T}^{(1)}$ in $\overline{m n}$ possono darsi due ipotesi:

$1^{a}$ ipotesi. $\xi$ è punto di discontinuita di $1^{\mathrm{a}}$ specie. Allora esiste un intoruo sinistro $\sigma$ di $\xi$, contenente una parte di $\mathcal{C O C}$, tale che, per tutti $\mathrm{i}$ valori di $x$ ad esso appartenenti, il rapporto incrementale $l$ di $F(x)$ su $\overline{x \xi}$ $\grave{e}$, in valore assoluto, $>\frac{1}{\varepsilon}(\varepsilon$ prefissato piccolo ad arbitrio). Si scelga un punto $c$ di $\mathcal{C} \mathscr{C}$, contenuto in $\sigma$, che non sia estremo sinistro di un intervallo

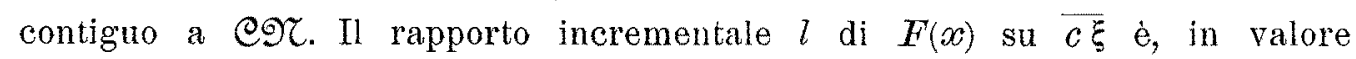
assoluto, $>\frac{1}{\varepsilon}$.

$2^{a}$ ipotesi. $\xi$ è punto di discontinuità di $2^{a}$ specie. Allora è possibile scegliere due punti $c, \bar{\xi}$ a sinistra di $\xi$ e in un intorno sinistro comunque piccolo di $\xi$, tali che $c$ appartenga a $\mathcal{C O}$ ma non sia estremo sinistro di un intervallo contiguo a $\mathcal{C O C}$ e che il rapporto incrementale di $F(x)$ su $\overline{c \bar{\xi}}$ sia, in valore assoluto, $>\frac{1}{\varepsilon}$.

Sia $\varepsilon_{1}, \varepsilon_{2}, \ldots, \varepsilon_{n}, \ldots$ una successione arbitrariamente prefissata di numeri positivi tendente a 0 . Siano $\xi_{1}, \xi_{2}, \ldots, \xi_{3}, \ldots 1$ punti di $\mathcal{Y}^{(1)}$ contenuti in $\overline{m n}$. Per ognuno di essi $\xi_{n}$, sia nella prima che nella seconda ipotesi, è possibile 
scegliere due punti $c_{\imath}, d_{n}$ contenuti entrambi nell'intervallo comume ai due intervalli $\overline{m n}, \xi_{n}-{\overline{\xi_{n}}}_{\xi_{n l}}$, il primo dei quali $c_{n z}$ appartenente a $\mathcal{C O C}$ ma non estremo sinistro di un intervallo contiguo a $\mathcal{C S}$, il secondo $d_{n}$ eventualmente (1) ipotesi) coincidente con $\xi_{n}$, tali che il rapporto incrementale $l_{n}$ di $F(x)$ su $\overline{c_{n} d_{n}}$ sia, in valore assoluto, $>\frac{1}{\varepsilon_{n}}$.

I segmenti $S_{n} \equiv \overline{c_{n s} d_{n}}$, per $n=1,2, \ldots$ sono nelle condizioni del teor. fondamentale (per il n.o 7 del $\$ 1$ ). Dunque esistono dei punti di Cor in $\overline{m n}$ per $\mathrm{i}$ quali è verificata almeno una delle due uguaglianze

$$
\bar{D}_{+} F(x)=+\infty, \quad D_{+} F(x)=-\infty \text {. }
$$

Ma ciò è assurdo perchè contrario all'ipotesi. Dunque $\mathscr{O}^{(1)}$ è non denso in tutto $\mathscr{K}$. Analogamente si dimostra che $\mathscr{T}^{(2)}$ è non denso in tutto $\mathscr{O}^{(1)}$, che $\mathscr{T}^{(3)}$ è non denso in tutto $\mathscr{V}^{(2)}, \ldots$, che $\mathscr{V}^{(n)}$ è non denso in tutto $\mathscr{T}^{(n-1)}, \ldots$

Le considerazioni del n. ${ }^{\circ}$ prec. intorno alla successione transfinita

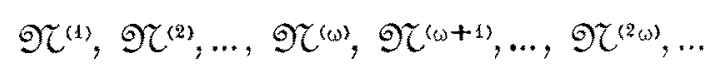

si ripetono identicamente punto per punto.

10. Teor. Se $\bar{D}_{+} F(x)$ e $D_{+} F(x)$ sono finite in tutti $\mathrm{i}$ punti di $\overline{a b}$ e se $\mathfrak{K}$ è un qualunque aggregato chiuso contenuto in $\overline{a b}$, l'insieme dei punti di $\mathscr{T}$ in cui $\bar{D}_{+} F(x)$ è non limitata superiormente $\left({ }^{20}\right)$ e l'insieme dei punti di $\mathscr{T}$ in cui $D_{+} F(x)$ è non limitata inferiormente sono ambedue chiusí $\left({ }^{\circledR 4}\right)$ $\mathrm{e}$ non densi in $\mathfrak{T K}$.

$\left({ }^{20}\right)$ Com'è noto una funzione $f(x)$ ¿̀ « limitata in un intervallo $\overline{a b}$ se esiste un numero positivo $M$ tale che $|f(x)|<M$ per tutti i punti $x$ di $\overline{a b}$.

Una funzione $f(x)$ è "limitata * in un punto $\bar{x}$ di $\overline{a b}$ se esiste un intorno di $\ddot{x}$ nel quale è limitata. $\dot{\mathrm{E}}$ "non limitata " in $\bar{x}$ nel caso contrario.

Una furizione $f(x) \dot{e}$ \&limitata in un punto $\bar{x}$ di un aggregato $\mathscr{N}$ contenuto in $\bar{a} b$, ri. spetto ad $\mathscr{O}$, se esiste un intorno $\overline{p q}$ di $\bar{x}$ e un numero $M$ tale che $|f(x)|<M$ per tutti punti comuni a $\overline{p q}$ e ad $\mathscr{T} \zeta$. $\dot{\mathrm{E}}$ in quest' ultimo senso che, nella presente dimostrazione, useremo l'espressione "funzione limitata in un determinato punto di un aggregato ».

(21) Che dehbano essere chinsi è evidente. Infatti se $\bar{x}$ è un punto di $\mathscr{9}$ in eui per es. $\bar{D}_{+} F(x)$ è limitata superiormente, cioè se $\bar{x}$ è interno a un intervallo nel quale

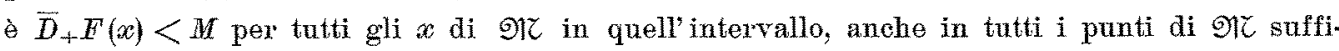
cientemente vicini a $\bar{x}$, poichè essi sono interni allo stesso intervallo, è $\bar{D}_{+} F(x)$ limitata superiormente. 
Drm. La dimostrazione si riallaccia da più parti al teor. fondamentale. Precisamente, limitandoci per es. alla $\bar{D}_{+} F(x)$, noi dimostreremo che, se $\overline{l m}$ è un qualunque intervallo contenuto in $\overline{a b}$ e contenente una parte di $\mathfrak{K}$, esistono un numero $M$ e un intervallo $\overline{r s}$ contento in $\overline{l m}$ e contenente una parte di $\mathscr{T}$ tali che, per tutti i punti $x$ comuni a $\mathfrak{N}$ e ad $\bar{r} s$, è $\bar{D}_{+} F(x)<M$.

Infatti osserviamo anzitutto che, poichè $\vec{D}_{+} F(x)$ e $\underline{D}_{+} F(x)$ sono finite in tutti i punti di $\overline{a b}$, l'aggregato $\mathfrak{C O}$ che si ottiene per chiusura dall'aggregato $\mathscr{O}$ dei punti di discontinuità di $F(x)$ è non denso in $\overrightarrow{a b}\left(\mathbf{n} .^{\circ} 5\right)$.

Se $\mathscr{T C}$ ha in $\overline{l m}$ un punto isolato $\bar{x}$, il segmento $\overline{r s}$ è un intorno contenente soltanto $\bar{x}$ e il numero $M$ è un qualunque numero $>\bar{D}_{+} F(\bar{x})$.

Se la parte di $\mathscr{T}$ che è contenuta in $\overline{l m}$ non è interamente contenuta in eor la dimostrazione è pure immediata. Infatti in questo caso esiste un intervallo $\overline{\alpha \beta}$ contenuto tanto in $\bar{l} m$ quanto in un intervalio contiguo a $\mathfrak{C O C}$ e contenente una parte di $\mathscr{K}$. In tutto l'intervallo $\overline{\alpha \beta}$ la funzione $F(x)$ è continua bilateralmente e l'esistenza del numero $M$ e dell'intervallo $\bar{r} s$ contenuto in $\overline{\alpha \beta}$ sono affermati da un corollario che il DenJor deduce dal teor. fondamentale (loc. cit., pag. 156).

Supponiamo dunque che la parte $\overline{\mathfrak{T}}$ di $\mathscr{T}$ contenuta in $\bar{l} \bar{m}$ sia perfetta e che sia altressí contenuta in $\mathcal{C Q T}$. Sia $\tau_{i} \equiv \overline{\mu_{i} \nu_{i}}$ un generico intervallo contiguo a $\overline{\mathfrak{9 K}}$.

In $\overline{l n}$ vi sono certamente dei punti $\mu_{i}$. Dico che i punti $\mu_{i}$ che sono punti di discontinuità per la $F(x)$ e che sono limiti verso sinistra di punti di discontinuità per la $F(x)$ costituiscono un insieme $Q$ non denso in $\overline{\mathscr{K}}$. Infatti nel caso contrario, ragionando per assurdo, basta ripetere una dimostrazione analoga a quella del $1 .^{\circ}$ 9. Per maggiore chiarezza la ripetiamo intieramente.

Sia, se possibile, $Q$ denso in $\overline{\mathscr{T}}$. Per ogni punto $\mu$ di $Q$ possono darsi due ipotesi:

$1^{a}$ ipotesi. $\mu$ è punto di discontinuità di $1^{\text {a }}$ specie. Allora esiste un intorno sinistro $\sigma$ di $\mu$, intieramente contenuto in $\overline{l m}$ e contenente una parte di $\overline{\mathfrak{K}}$, tale che, per tutti i valori di $x$ ad esso appartenenti, il rapporto incrementale $l$ di $F(x)$ su $\overline{x \mu}$ è, in valore assoluto, $>\frac{1}{\varepsilon}(\varepsilon$ prefissato piccolo ad arbitrio). Si scelga un punto $c$ di $\overline{\mathscr{T K}}$, contenuto in $\sigma$, che non sia estremo sinistro di un intervallo contiguo a $\overline{\mathscr{T}} \bar{K}$. Il rapporto incrementale $l$ di $F(x)$ su $\overline{c \mu} \dot{e}$, in valore assoluto, $>\frac{1}{\varepsilon}$. 
$2^{a}$. ipotesi. $\mu$ è pnnto di discontinuita di $2^{\text {a }}$ specie. Allora è possibile scegliere due punti $c, \mu^{\prime}$ a sinistra di $\mu$ e in un intorno sinistro comunque piccolo di $\mu$, tali che $c$ appartenga ad $\overline{\mathfrak{T}}$ ma non sia estremo sinistro di un intervallo contiguo a $\overline{\mathfrak{K}}$, e che il rapporto incrementale di $F(x)$ su $\bar{c} \overline{\mu^{\prime}}$ sia, in valore assoluto, $>\frac{1}{\varepsilon}$.

Per ogni punto $\mu_{i}$ di $Q$, sia nella $1^{\text {a }}$ che nella $2^{a}$ ipotesi, è possibile scegliere due punti $c_{i}, d_{i}$ contenuti entrambi nell'intervallo comune ai due intervalli $\overline{l n}, \mu_{i}-\overline{\varepsilon_{i} \mu_{i}}$ (essendo $\varepsilon_{1}, \varepsilon_{2}, \ldots, \varepsilon_{i}, \ldots$ una successione arbitrariamente prefissata di unmeri positivi tendente a $O$ ), il primo dei quali $c_{i}$ appartenente ad $\overline{\mathscr{T R}}$ ma non estremo sinistro di un intervallo contiguo ad $\overline{\mathscr{T R}}$, il secondo $d_{i}$ eventualmente ( ${ }^{a}$ ipotesi) coincidente con $\mu_{i}$, tali che il rapporto incrementale $l_{i}$ di $F(x)$ su $\overrightarrow{c_{i} d_{i}}$ sia, in valore assoluto, $>\frac{1}{\varepsilon_{i}}$.

I segmenti $S_{i} \equiv{\overline{c_{i}}}_{i}$, per $i=1,2, \ldots$ sono nelle condizioni del teorema fondamentale (per il $\$ 1, n{ }^{\circ}$ 7). Dunque esistono dei punti di $\overline{\text { TK }}$ per i quali è verificata almeno una delle due uguaglianze

$$
\bar{D}_{+} F(x)=+\infty, \quad \underline{D}_{+} F(x)=-\infty \text {. }
$$

Ma ciò è assurdo perchè contrario all'ipotesi. Dunque $Q$ è non denso in $\overline{\mathfrak{Y T}}$.

Sia $l^{\prime} m^{\prime}$ un intercallo contenuto in $\overline{l m}$, contenente una parte $\overline{\mathscr{N}} \bar{\kappa}^{\prime}$ di $\overline{\mathscr{K}}$ ma nessun punto di $Q$. Dico che $\overline{\gamma s}$ esiste entro $\overline{l^{\prime} m^{\prime}}$. Ragioniamo infatti per assurdo e supponiamo, se possibile, che $\bar{D}_{+} F(x)$ sia non limitata superiormente in ogni punto di $\overline{\mathscr{9 K}}$ (rispetto a $\overline{\mathscr{T}} \overline{\mathcal{C}^{\prime}}$ ). Segnamo in $\overline{\mathscr{9} \bar{K}^{\prime}}$ una successione di punti $\gamma_{1}, \gamma_{2}, \gamma_{3}, \ldots$ ovunque densa in $\overline{\mathscr{9 K}}$. Esiste nell' intorno $\gamma_{1}-\overline{\varepsilon_{1} \gamma_{1}}-1-\varepsilon_{1}$ dal punto $\gamma_{1}$ un punto $p_{1}$ di $\overline{\mathscr{N} \overline{\mathbb{C}}^{\prime}}$ tale che $\bar{D}_{+} F\left(p_{1}\right)>\frac{2}{\varepsilon_{-j}}$ e quindi esiste un punto $q_{1}$ alla destra li $p_{1}\left(q_{1}\right.$ non appartiene necessariamente nè a $\overline{\mathscr{G C}}$ nè a egr) tale che $\frac{F\left(q_{1}\right)-F\left(p_{1}\right)}{q_{1}-p_{1}}>\frac{1}{\varepsilon_{1}}$ e che $q_{1}-p_{1}<\varepsilon_{1}$. Esiste nell' intorno $\gamma_{2}-\overline{\varepsilon_{2} \gamma_{2}}+\varepsilon_{2}$ del punto $\gamma_{2}$ un punto $p_{2}$ di $\overline{\mathscr{N} \tau^{\prime}}$ tale che $\bar{D}_{+} F\left(p_{2}\right)>\frac{2}{\varepsilon_{2}}$ e quindi esiste un punto $q_{2}$ alla destra di $p_{2}$ tale che $\frac{F\left(q_{z}\right)-F\left(p_{2}\right)}{q_{2}-p_{2}}>\frac{1}{\varepsilon_{2}}$ e che $q_{2}-p_{2}<\varepsilon_{2}$, ecc. In generale esiste nell'intorno $\gamma_{r}-\overline{\varepsilon_{r} \gamma_{r}}+\varepsilon_{r}$ del punto $\gamma_{r}$. 
un punto $p_{r}$ di $\overline{\mathfrak{O C}^{\prime}}$ tale che $\bar{D}_{+} F\left(p_{r}\right)>\frac{2}{\varepsilon_{r}}$ e quindi esiste in punto $q_{r}$ alla destra di $p_{r}$ tale che $\frac{F\left(q_{r}\right)-F\left(p_{r}\right)}{q_{r}-p_{r}}-\frac{1}{\varepsilon_{r}}$ e che $q_{r}-p_{r}<\varepsilon_{r}$.

I segmenti $T_{r} \equiv{\overline{p_{r} q_{r}}}$, per $r^{*}=1,2, \ldots$ sono nelle condizioni del teorema fondamentale rispetto ad $\overline{\mathscr{N}}$ '. Infatti se $p_{r}$ è estremo sinistro di un intervallo contiguo ad $\overline{\mathfrak{I R}^{r}}, p_{r}$, è limite verso sinistra di punti di discontinuita (perchè $\overline{\mathscr{T}} \overline{\mathcal{C}}^{\prime}$ è intieramente contenuto in $\mathscr{C} \mathcal{K}$ ); dunque $p_{r}$. non è punto di discontinuità. Se $p_{r}$. non è estremo sinistro di un intervallo contiguo ad $\overline{\mathscr{K}} \bar{C}^{\prime}$, esiste un intorno destro di $p_{r}$ che contiene punti di $\overline{\mathscr{N}}{ }^{\prime}$ (vedi il $\& 1$, n. ${ }^{\circ} 7$ ).

Dunque esistono dei punti di $\overline{\mathscr{T K}}$ per i quali è verificata almeno una delle due uguaglianze

$$
\bar{D}_{+} F(x)=+\infty, \quad \underline{D}_{+} F(x)=-\infty,
$$

il che è nuovamente assurdo, come sopra.

Cosi il teorema è completamente dimostrato.

11. Il teorema fondamentale si potrebbe applicare, coi dovuti riguardi, anche dalla banda sinistra $\left({ }^{22}\right)$. Ci limitiamo a dimostrare direttamente il seguente

Teor. Se l'aggregato

$$
\mathscr{O} \equiv d_{1}, d_{2}, \ldots, d_{n}, \ldots
$$

dei punti di discontinuità di $F(x)$ è denso in tutto $\overline{a b}$, allora l'insieme de $i$ punti di continuitá di $F(x)$ in $\overline{a b}$, nei quali è verificata almeno una delle due relazioni

$$
\bar{D}_{-} F(x)=+\infty, \quad D_{-} F(x)=--\infty
$$

è denso in tutto $\overline{a b}$.

DrM. Sia $\varepsilon_{1}, \varepsilon_{2} \ldots, \varepsilon_{i n}, \ldots$ una successione di numeri positivi tendente a 0 . Sia $d_{r_{1}}$ un punto qualunque di $\mathscr{Q}$. Alla sinistra di $d_{r_{1}}$ esiste certamente un punto $c_{r_{1}}$, appartenente $\overline{a b}$, tale che $d_{r_{2}}-c_{r_{3}}<\varepsilon_{1}$ e che $\frac{\left|F\left(d_{r_{1}}\right)-F\left(c_{r_{1}}\right)\right|}{d_{r_{1}}-c_{r_{1}}}>\frac{2}{\varepsilon_{1}}$, e ció per la discontinuitả verso sinistra di $F(x)$ in $d_{r_{1}}$. Alla destra di $d_{r_{1}}$ esiste certamente un intorno $\sigma_{1}$ di ampiezza minore di $\varepsilon_{1}$, intieramente contenuto in $\overline{a b}$, tale che $\frac{\left|\boldsymbol{F}(x)-F\left(c_{r_{1}}\right)\right|}{x-c_{r_{1}}}>\frac{1}{\varepsilon_{1}}$, per tutti i valori di $x$ ad esso apparte-

$\left({ }^{2}\right)$ S'intende riguardo ai punti di continuità, poichè soltanto in questi sono definiti i numeri derivati a sinistra. 
nenti. Sia $d_{r_{3}}$ un punto qualunque, di $\mathscr{\mathcal { C }}$ in $\sigma_{1}$, distinto da $d_{r_{1}}\left(r_{2}>r_{1}\right)$. Alla sinistra di $d_{r_{2}}$ esiste $u n$ punto $c_{r_{2}}$ appartenente ad $\overline{a b}$, tale che $d_{r_{2}}-c_{r_{2}} \div \varepsilon_{2}$ e che $\frac{\left|F\left(d_{r_{2}}\right)-F\left(c_{r_{2}}\right)\right|}{d_{r_{3}}-c_{r_{a}}}>\frac{2}{\varepsilon_{z}}$. Alla destra di $d_{r_{a}}$ esiste un intorno $\sigma_{2}$ di ampiezza minore di $\varepsilon_{2}$, intieramente contenuto in $\sigma_{1}$ tale che $\frac{\left|F(x)-F\left(c_{r_{2}}\right)\right|}{x-c_{r_{2}}}>\frac{1}{\varepsilon_{2}}$, per tutti i valori di $x$ ad esso appartenenti. Sia $d_{r_{3}}$ un punto qualunque di $\mathscr{N}$ in $\sigma_{z}$, distinto da $d_{r_{z}}\left(r_{3}>r_{2}\right)$. Cosi si prosegue indefinitamente: in generale, essendosi scelto $d_{r_{n}}$ che segue $d_{r_{n-1}}$ nell' ordinamento di $\mathscr{T}$, si osserverà che alla sinistra di $d_{r_{n}}$ esiste un punto $c_{r_{n}}$ appartenente ad $\overline{a b}$, tale che $d_{r_{n}}-c_{r_{n}}<\varepsilon_{n}$ e che $\frac{\left|\boldsymbol{F}\left(d_{r_{n}}\right)-\boldsymbol{F}\left(c_{r_{n}}\right)\right|}{d_{r_{n}}-c_{r_{n}}}>\frac{2}{\varepsilon_{n}}$. Alla destra di $d_{r_{n}}$ esiste un intorno $\sigma_{n}$ di ampiezza minore di $\varepsilon_{n}$, intieramente contenuto in $\sigma_{n-1}$, tale che $\frac{\left|F(x)-F\left(c_{r_{n}}\right)\right|}{x-c_{r_{n}}}>\frac{1}{\varepsilon_{n}}$ per tutti i valori di $x$ ad esso appartenenti. Sia $d_{\psi_{n+1}+1}$ un punto qualunque di $\mathscr{O}$ in $\sigma_{n}$, distinto da $d_{r_{n}}\left(r_{n+1}>r_{n}\right)$.

Si può sempre scegliere la successione crescente

$$
d_{r_{1}}, d_{r_{2}}, \ldots, d_{r_{n}}, \ldots
$$

in modo che abbia per limite un punto di continuita. In quel punto esistono numeri derivati sinistri, e precisamente deve essere verificata almeno una delle due relazioni enunciate. Inoltre, data la scelta arbitraria di $d_{r_{\text {s }}}$ e del suo intorno destro $\sigma_{1}$, tali punti di continuità sono ovunque densi in $\bar{a} \bar{b}\left({ }^{23}\right)$. c. d. d.

("3) Il procedimento del $n .^{\circ} 11$ è simile a quello del $n .^{\circ} 5$, ma ben più di quello sembra far uso del postulato di ZERMELo, onde può far dubitare della sua correttezza. Vale la pena che c'indugiamo per mostrare la possibilità di liberarnelo.

Scelto ad arbitrio $d_{r_{1}}$ in $\mathscr{K}$, può scegliersi per $c_{r_{1}}$ il primo punto di $\mathscr{O}$ medesimo soddisfacente alle condizioni volute. Tale primo punto esiste certamente: infatti sia $x_{0}$ un qualunque punto (ciò̀ anche non appartenente ad $\mathscr{K}$ ) alla sinistra $d i{ }_{r_{1}}$, appartenente ad $\overrightarrow{a b}$, tale che $d_{r_{1}}-x_{0}<\varepsilon_{1}$ e ohe $\frac{\left|\vec{F}\left(d r_{1}\right)-F\left(x_{0}\right)\right|}{d r_{1}-x_{0}}>\frac{3}{\varepsilon_{1}} \cdot x_{0}$ esiste certamente ed esiste un intorno destro di $x_{0}$, intieramente contenuto in $\overline{x_{4} d_{r_{1}}}$, tale che $\frac{\left|F\left(d r_{1}\right)-F(x)\right|}{d r_{1}-x}>\frac{2}{\varepsilon_{1}}$ per tutti i valori di $x$ ad esso appartenenti, quindi anche per tutti $i$ punti di discontinuità ad esso appartenenti. Analogamente possono regolarizzarsi le scelte dei successivi $c_{r_{2}}, c_{r_{3}}, \ldots, c_{r_{n}}, \ldots$

Anche la scelta dei punti di discontinuità $d_{r_{3}}, d_{r_{3}}, \ldots, d_{r_{n}}, \ldots$ può facilmente regolariz. zarsi in modo da soddisfare alle condizioni volute. Basta per es. porre la condizione

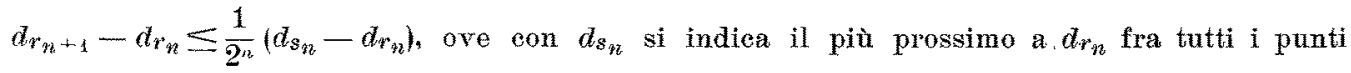
di $\mathscr{O} \mathcal{C}$ che hanno indice $\left\langle r_{n}\right.$ e che sono $>d_{r_{n}}$. Infatti se la successione $d_{r_{1}}, d_{r_{2}}, \ldots, d_{r_{n}}, \ldots$ costruita con questa condizione tendesse ad un punto $d_{k}$ di discontinuità, basterebbe consi- 
Nella dimostrazione non ci siamo indugiati sopra i dettagli, per non ripeterci. Per essi rimandiamo al $\$ 1$, n. ${ }^{\circ} 2$.

12. I procedimenti dei $n .{ }^{i} 5$ e 11 si possono alternare in modo analogo a quanto si è fatto al $\S 1, n^{\circ}$ 3. Riassumendo allora entrambi $i 1^{i} 5$ ed 11 , si ottiene il seguente notevole

Teor. Se $\mathscr{K}$ è denso in un segmento $\overline{m n}$ parziale di $\bar{a} \bar{b}$ (eventualmente in tutto $\overline{a b}$ ) l'aggregato dei punti $x$ di continuità in cui da ogni parte almeno uno dei numeri derivati estremi è infinito, è denso in $\overline{m n}$.

Dim. A ciascuno dei punti $d_{1}, d_{2}, \ldots, d_{n}, \ldots$ dell' aggregato $\mathscr{T}$ facciamo corrispondere un punto $c_{j}(j=1,2, \ldots, n, \ldots)$ secondo la regola indicata al $n .{ }^{\circ} 5$.

Alla destra del punto $c_{r_{1}}\left(r_{1}\right.$ è un indice arbitrario) esiste certamente $u$ intorno $\sigma_{1}$ di ampiezza minore di $\varepsilon_{1}$, intieramente contenuto in $\overline{a b}$, tale che $\frac{\left|F\left(d_{r_{1}}\right)-F(x)\right|}{d_{r_{1}}-x}>\frac{1}{2 \varepsilon_{1}}$ per tutti i valori di $x$ ad esso appartenenti. Sia $d_{r_{3}}$ un punto qualunque di $\mathscr{K}$ in $\sigma_{1}$, distinto da $c_{r_{1}}\left(r_{2}>r_{1}\right)$. Alla destra di $d_{r_{2}}$ esiste certamente un intorno $\sigma_{2}$, di ampiezza minore di $\varepsilon_{2}$, intieramente contenuto in $\sigma_{1}$, tale che $\frac{\left|F(x)-F\left(c_{r_{3}}\right)\right|}{x-c_{r_{2}}}>\frac{1}{2 \varepsilon_{2}}$ per tutti i valori di $x$ ad esso appartenenti. Sia $c_{r_{3}}$ un punto $c_{j}$ in $\sigma_{2}$, distinto da $d_{r_{2}}\left(r_{3}>r_{2}\right)$. Cosi si prosegua indefinitamente: in generale, essendosi scelto $c_{r_{2 n-1}}$, si osserverà che alla destra di $c_{r z_{n-1}}$ esiste un intorno $\sigma_{2 n-1}$ di ampiezza $<\varepsilon_{2 n-1}$, intieramente contenuto in $\sigma_{2 n-2}$, tale che $\frac{\left|F\left(d_{r_{2 n-1}}\right)-F(x)\right|}{d_{r_{2 n-1}}-x}>\frac{1}{2 \varepsilon_{2 n-1}}$ per tutti i valori di $x$ ad esso appartenenti. Sia $d_{r_{2 n}}$ un punto qualunque di $\mathscr{T}$ in $\sigma_{2 n-1}$, distinto da $c_{r_{2 n-1}-1}\left(r_{2 n}>r_{2 n-1}\right)$. Alla destra di $d_{r_{2 n}}$ esiste un intorno $\sigma_{2 n}$ di ampiezza $<\varepsilon_{2 n}$, intieramente contenuto in $\sigma_{2 n-1}$, tale che $\frac{\left|F(x)-F\left(c_{r_{2 n}}\right)\right|}{x-c_{r_{2 n}}}>\frac{1}{2 \varepsilon_{2 n}}$

derare il più piccolo intiero $n$ tale che $r_{n}>k$. Sarebbe $d_{r_{n}}<d_{k}$, ma

$$
\begin{aligned}
& d_{r_{n+1}}-d_{r_{n}} \leq \frac{1}{2^{n}}\left(d_{k}-d_{r_{n}}\right) \\
& d r_{n+2}-d r_{n} \leq \frac{1}{2^{n}}\left(d_{k}-d_{r_{n}}\right)+\frac{1}{2^{n+1}}\left(d_{k}-d_{r_{n+1}}\right)<\left(\frac{1}{2^{n}}+\frac{1}{2^{n+1}}\right)\left(d_{k}-d_{r_{n}}\right) . \\
& d_{r_{n+3}}-d_{r_{n}}<\left(\frac{1}{2^{n}}+\frac{1}{2^{n+1}}+\frac{1}{2^{n+2}}\right)\left(d_{k}-d_{r_{n}}\right) . \\
& \ldots \ldots \ldots \ldots \ldots \ldots
\end{aligned}
$$

e al limite

Assurdo.

$$
d_{k}-d_{r_{n}}<\frac{1}{2^{n}}\left(1+\frac{1}{2}+\frac{1}{4}+\ldots\right)\left(d_{k}-d_{r_{n}}\right)=\frac{1}{2^{n-1}}\left(d_{n}-d_{r_{n}}\right)
$$


per tutti i valori di $x$ ad esso appartenenti. Sia $c_{r_{2 n+1}}$ un punto $c_{j}$ in $\sigma_{2 n}$, distinto da $d_{r_{2 n}}\left(r_{2 n+1}>r_{z n}\right)$.

Si puó sempre scegliere la successione crescente

$$
c_{r_{1}}, d_{r_{2}}, c_{r_{s}}, d_{r_{4}}, \ldots, c_{r_{2,2}-1}, d_{r_{2 n}}, c_{r_{2 n+1}}, \ldots
$$

in modo che abbia per limite un punto di continuità. In quel punto esistono i derivati destri e i derivati sinistri e precisamente deve valere la proposizione enunciata. Inoltre, data la scelta arbitraria di $c_{r_{1}}$ e del suo intorno destro $\sigma_{1}$, tali punti di continuitá sono ovunque densi in $\overline{a b}\left({ }^{24}\right)$.

13. Nella dimostrazione del teor. del $1 .{ }^{\circ} 11$, l'ipotesi che $\mathscr{C}$ sia denso in $\overline{a b}$ può sostituirsi con altra equivalente: ogni punto di $\mathscr{\mathscr { C }}$ è limite verso la sua destra di punti di $\mathscr{T}$, oppure: i punti di $\mathscr{\mathscr { C }}$ che sono limiti verso la loro destra di punti di $\mathscr{K}$ costituiscono un aggregato ovunque denso in $\mathscr{K}\left({ }^{2 \check{y}}\right)$. La tesi che allora si dimostra col procedimento del $n .^{\circ} 11$ è: i punti dí continuità in cui è verificata almeno una delle due relazioni $\bar{D} \_F(x)=+\infty$, $D_{-} F(x)=-\infty$, costituiscono un aggregato ovunque denso in $\mathscr{\mathcal { K }}\left({ }^{26}\right)$.

14. Terminiamo con alcuni interessanti esempi:

10) Funzione $F(x)$ derivabile ovunque verso destra nell' intervallo $\overline{0} \overline{1}$. L'aggregato $\mathscr{T}$ dei punti di discontinuità è costituito dagli estremi destri degl'intervalli contigui al noto aggregato perfetto non denso di CaNTor $\left({ }^{27}\right)$. In tutti i punti dell'aggregato perfetto di CaNTor la derivata destra è nulla, in tutti gli altri punti è $>0$. La funzione si costruisce nel modo seguente:

si pone $F(x)=C$ (costante arbitrariamente prescelta) in tutti i punti dell' aggregato di CANTOR;

se $\overline{\alpha_{n} \beta_{n}}$ è un generico intervallo contiguo, per ogni $x$ interno ad esso si pone $F(x)=C+\left(x-\alpha_{n}\right)^{2}$.

Per ogni punto $\bar{x}$ dell' aggregato di CANToR è, se $x>\bar{x}$,

$$
C \leq F(x) \leq C+(x-\bar{x})^{2} .
$$

(24) Vedi la nota $\left({ }^{23}\right)$.

(25) Vedi il $\$ 1$, n. $^{\circ} 7$, e il $\$ 2, n .^{\circ} 8$.

(*6) Cioè entro ogni segmento $\overline{m n}$ contenente punti di $\mathscr{O C}$ esistono punti di continuità $x$ in cui è verificata almeno una delle due relazioni $\bar{D} \ldots(x)=+\infty, D_{-} F(x)=-\infty$.

(27) Per la definizione e le proprietà di questo aggregato vedi ad es. Lebescun, Lecons sur l'intégration, 1928, pag. 27, oppure TonelLI, Calcolo delle Variazioni, 1921, vol. I, pag. 108, oppure Denjox, loe. eit., pag. 121. 
I punti dell'aggregato di Cantor che sono punti di continuità e nei quali è $D_{-} F(x)=-\infty$ costituiscono un aggregato ovunque denso nell' aggregato di Cantor (per il $10^{\circ} 13$ ). Evidentemente in tutti questi punti è però anche $\bar{D}_{-} F(x)=0$.

15. $\left.2^{\circ}\right)$ Funzione $F(x)$ continua ovunque verso destra nell' intervallo $\overline{01}$. L'aggregato $\mathscr{R}$ dei punti di discontinuità è quello dei punti di $\overline{01}$ che in

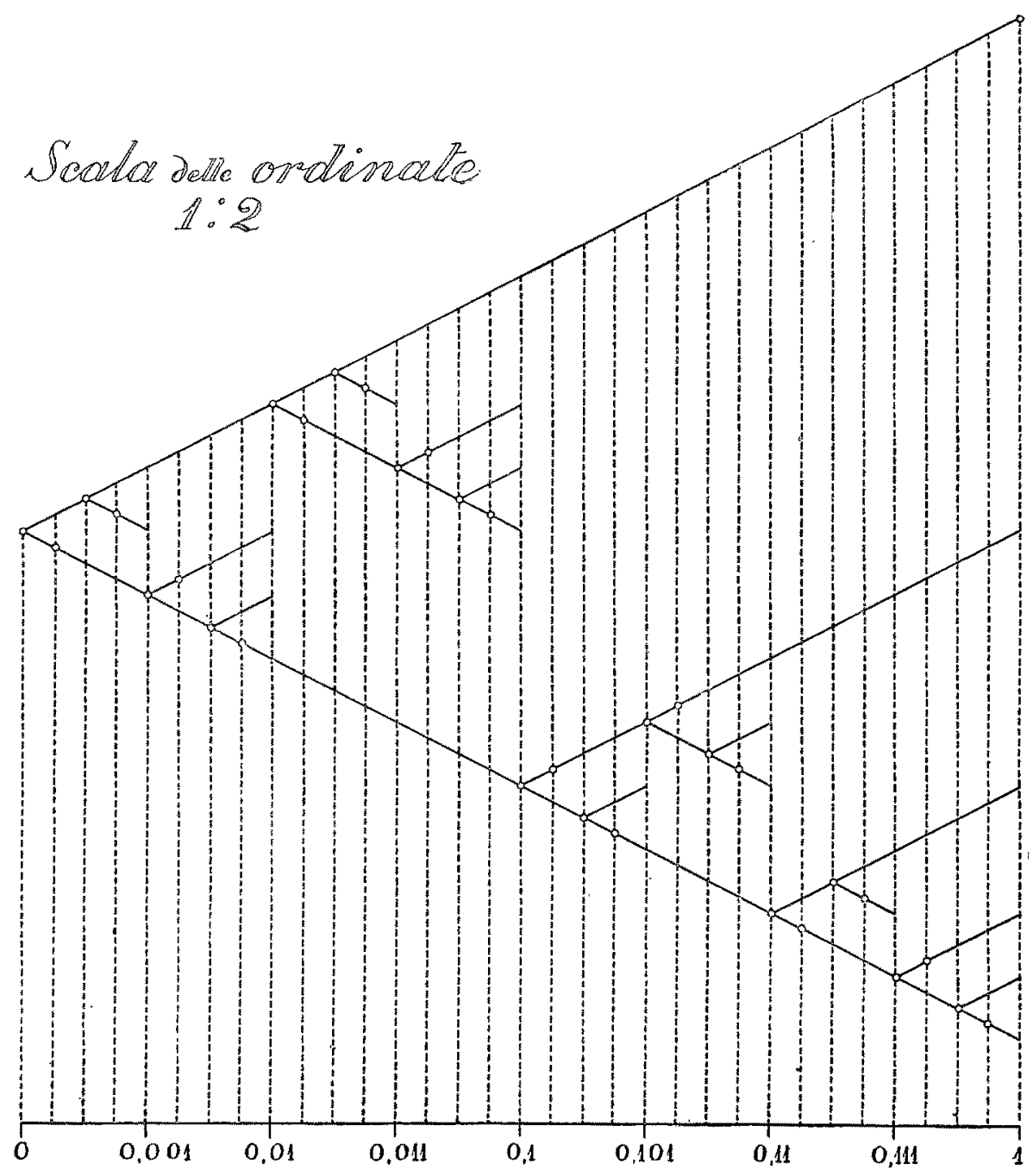

numerazioue binaria si rappresentano con un numero finito di cifre dopo la virgola (cifre 0 e 1 ) ed è denso in tutto $\overline{01}$. In tutti i punti di $\mathscr{\mathscr { K }}$ è $\bar{D}_{+} F(x)=+1$, 
$D_{+} F(x)=-1$. La figura qui disegnata nè dà una rappresentazione cartesiana approssimata.

La funzione si costruisce nel modo seguente. Si assegnano ad $F(0)$ e ad $F(1)$ valori arbitrari. Indi si pone

$$
\left\{\begin{array}{l}
F\left(\frac{1}{4}\right)=F(0)+\frac{1}{4}, \quad F\left(\frac{1}{16}\right)=F(0)+\frac{1}{16}, \ldots, \quad F\left(\frac{1}{2^{2 n}}\right)=F(0)+\frac{1}{2^{2 n}}, \ldots \\
F\left(\frac{1}{2}\right)=F(0)-\frac{1}{2}, \quad F\left(\frac{1}{8}\right)=F(0)-\frac{1}{8}, \ldots, \quad F\left(\frac{1}{2^{2 n-1}}\right)=F(0)-\frac{1}{2^{2 n-1}}, \ldots
\end{array}\right.
$$

Si è cosi definita la funzione in un primo aggregato di punti $Q(0)$ aventi per limite il punto $O$.

Si consideri l'aggregato $Q\left(\frac{1}{2}\right)$ simile $\left({ }^{28}\right)$ a $Q(0)$ contenuto in $\overline{\frac{1}{2}} 1$ e si definisca la funzione $F(x)$ per tutti i valori di $Q\left(\frac{1}{2}\right)$ in modo che la differenza $F(x)-F\left(\frac{1}{2}\right)$ stia alla corrispondente differenza già definita $F(x)-F(0)$ nel rapporto di similitudine $\left(=\frac{1}{2}\right)$ che intercede fra $Q\left(\frac{1}{2}\right)$ e $Q(0)$.

Si consideri l'aggregato $Q\left(\frac{1}{4}\right)$ simile a $Q(0)$ contenuto in $\frac{11}{4} \frac{1}{2}$ e si definisca la funzione $F(x)$ per tutti i valori di $Q\left(\frac{1}{4}\right)$ in modo che la differenza $F(x)-F\left(\frac{1}{4}\right)$ stia alla corrispondente differenza già definita $F(x)-F(0)$ nel rapporto di similitudine $\left(=\frac{1}{4}\right)$ che intercede fra $Q\left(\frac{1}{4}\right)$ e $Q(0)$.

Così si prosegue: in generale si consideri l'aggregato $Q\left(\frac{k}{2^{n}}\right)$ simile a $Q(0)$, definito per ogni numero $\frac{k}{2^{n}}\left(n=1,2,3, \ldots ; k<2^{n}\right)$, contenuto in $\frac{\bar{k} k}{2^{n}} \frac{k}{2^{n}}$, e si definisca la funzione $F(x)$ per tutti i valori di $Q\left(\frac{k}{2^{n}}\right)$ in modo che la dif-

(28) Cioè tale che la distanza mntua di tutti $i$ suoi punti e dagli estremi dell'inter* vallo $\frac{\overline{1}}{2}$ in eui sono contenuti, stia alla corrispondente distanza dei punti di $Q(0)$ in $\overline{01}$, nel rapporto di similitudine $\left(-\frac{1}{2}\right)$ che intercede fra gl' intervalli $\overline{\frac{1}{2}} 1$ e $\overline{01}$. 
ferenza $F(x)-F\left(\frac{k}{2^{n}}\right)$ stia alla corrispondente differenza già definita $F(x)-F(0)$ nel rapporto di similitudine $\left(=\frac{1}{2^{n}}\right)$ che intercede fra $Q\left(\frac{k}{2^{n}}\right)$ e $Q(0)$.

É evidente che la successione indefinita di queste operazioni fornisce la definizione della $F(x)$ in tutti i punti dell'aggregato $\mathscr{T}$. Uno sguardo alla figura prova poi che tutti i punti dell'aggregato $\mathscr{V}$ sono realmente punti di discontinuità; che in essi la $F(x)$ è continua verso destra e che è

$$
\bar{D}_{+} F(x)=+1, \quad D_{+} F(x)=-1 .
$$

In tutti gli altri punti di $\overline{01}$ la $F(x)$ è definita per continuita. Ciò è possibile: sia infatti $\xi$ un punto di $\overline{01}$ non appartenente ad $\mathscr{N}$, e sia

$$
\xi=\frac{\alpha_{1}}{2}+\frac{\alpha_{2}}{2^{2}}+\frac{\alpha_{3}}{2^{3}}+\ldots+\frac{\alpha_{n}}{2^{n}}+\ldots \quad\left(\alpha_{n}=\left\{\begin{array}{l}
0 \\
1
\end{array}\right)\right.
$$

la sua espressione in numerazione binaria. In tutti i punti di $\mathscr{\mathcal { C }}$ che si trovano nell'intervallo $\frac{\alpha_{1}}{2} \frac{\alpha_{1}+1}{2}$ la $F(x)$ assume valori contenuti nell'angolo avente vertice in $\left[\frac{\alpha_{1}}{2}, F\left(\frac{\alpha_{1}}{2}\right)\right]$, ampiezza $=\frac{\pi}{2}\left({ }^{29}\right)$ e bisettrice orizzontale verso destra. In tutti i punti di $\mathscr{X}$ che si trovano nell' intervallo $\frac{\alpha_{1}}{2}+\frac{\alpha_{\frac{2}{2}}}{2^{2}} \frac{\alpha_{1}}{2}+\frac{\alpha_{2}+1}{2^{2}}$ la $F(x)$ assume valori contenuti nell' angolo avente vertice in $\left[\frac{\alpha_{1}}{2}+\frac{\alpha_{2}}{2^{2}}\right.$, $\left.F\left(\frac{\alpha_{1}}{2}+\frac{\alpha_{2}}{2^{2}}\right)\right]$, ampiezza $=\frac{\pi}{2}$ e bisettrice orizzontale verso destra. In generale: in tutti $\mathrm{i}$ punti di $\mathscr{\mathcal { C }}$ che si trovano nell'intervallo

$$
\frac{\alpha_{1}}{2}+\frac{\alpha_{2}}{2^{2}}+\ldots+\frac{\alpha_{n}}{2^{n}} \frac{\alpha_{1}}{2}+-\frac{\alpha_{2}}{2^{2}}+\ldots+\frac{\alpha_{n-1}}{2^{n-1}}+\frac{\alpha_{n}+1}{2^{n}}
$$

la $F(x)$ assume valori contenuti nell' angolo avente vertice in

$$
\left[\frac{\alpha_{1}}{2}+\frac{\alpha_{2}}{2^{2}}+\ldots+\frac{\alpha_{n}}{2^{n}}, \quad F\left(\frac{\alpha_{1}}{2}+\frac{\alpha_{2}}{2^{2}}+\ldots+\frac{\alpha_{n}}{2^{n}}\right)\right]
$$

impiezza $=\frac{\pi}{2}$ e bisettrice orizzontale verso destra.

(29) Nella figura, per economia di spazio, si è fatto uso di scale diverse per le ascisse e per le ordinate. 
Poichè questa successione d'intervalli aperti verso destra non si arresta a nessun indice finito, ma prosegue indefinitamente avendo per limite il punto $\xi$, è chiaro che esiste il $\lim _{x \rightarrow \xi} F(x)$ ed è lecito quindi completare la definizione della fumzione ponendo $F(\xi)=\lim _{x \rightarrow \xi} F(x)$.

Applicando il ragionamento del n. ${ }^{\circ} 12$ si puó poi vedere che i punti di $\overline{01}$, non appartenenti ad $\mathscr{\mathscr { C }}$, in cui $\mathrm{i}$ quattro numeri derivati estremi di $F(x)$ sono tutti infiniti, costituiscono un aggregato denso in $\overline{01}$.

16. $3^{\circ}$ ) Una classe assai estesa di funzioni continue verso destra si puó definire nel modo seguentc.

Prefissato l'aggregato $\mathscr{X}$ dei punti di discontinuita

$$
a_{1}, a_{2}, \ldots, a_{n}, \ldots,
$$

denso in tutto un intervallo $\overline{a b}$, facciamo corrispondere biunivocamente ad ogni suo elemento il termine con lo stesso indice di una serie $S=u_{1}+u_{2}+\ldots$ a termini positivi, convergente.

Sia $x$ un punto di $\overline{a b}$. Se $x$ è un $a_{r}$, estraggo da $\mathscr{T}$ un gruppo di termini (necessariamente in numero finito) aventi indici $\leqslant r$ e susseguentisi sul segmento $\overline{a b}$ da sinistra verso destra Eseguisco l'estrazione con la legge seguente: $a_{m_{1}}$ è il primo termine di $\mathscr{O}$ tale che

$$
m_{1} \leq \gamma^{\prime}, \quad a_{m_{1}} \leq a_{\nu},
$$

$a_{m_{\mathrm{a}}}$ è il primo termine di $\mathscr{N}$ tale che

$$
m_{1}<m_{2} \leq r, \quad a_{m_{1}}<a_{m_{2}} \leq a_{r},
$$

$\ldots$, arrestando il procedimento al primo termine per cui è $m_{n}=r\left({ }^{30}\right)$.

Se $x$ non è un $a_{r}$, estraggo con la medesima legge da $\mathscr{R}$ un gruppo di termini (necessariamente in numero infinito) susseguentisi da sinistra verso destra sul segmento $\overline{a b}$, aventi indici ordinatamente crescenti e tendenti al limite $x$. Nell' un caso e nell' altro pongo

$$
F(x)=u_{m_{1}}+u_{m_{\mathrm{s}}}+u_{m_{\mathrm{s}}}+\ldots
$$

cioè definisco $F(x)$ come somma rispettivamente di un numero finito, oppure di una serie, di termini estratti (in ordine crescente) dallat serie $S$.

$\left.{ }^{(30}\right)$ Se fosse, in particolare, $m_{1}=r$ il gruppo estratto si ridurrebbe al solo termine $a_{m_{1}} \equiv a_{r}$. 
La funzione $F(x)$ cosi definita è continua verso destra in tutto $\bar{a} b$. Infatti l'incremento destro relativo ad un punto $x$ qualunque è della forma

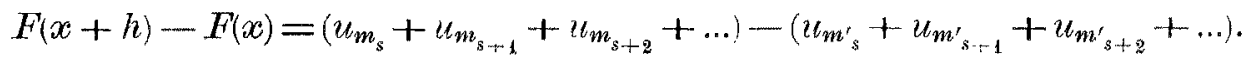

In questa espressione, se tanto $x$ che $x+h$ non appartengono ad $\mathscr{O C}$, minuendo e sottraendo nel secondo membro sono delle serie ed è $x<a_{m s}<$ $\left\langle x+h, m_{s}^{\prime}\right\rangle m_{s}$. Se $x$ appartiene ad $\mathscr{O}$, il sottraendo è una somma di un numero finito di termini che puó eventualmente scomparire del tutto (come avviene effettivamente da un certo punto in poi quando $h$ tende a zero). Se $x+h$ appartiene ad $\mathscr{T}$, il minuendo è una somma di un numero finito di termini, eventualmente ridotta al solo primo termine $u_{m s}$. In ogni caso, fissato arbitrariamente un intiero $\mu$, basta prendere $h$ minore della distanza di $x$ da ciascuno dei punti $a_{1}, a_{2}, \ldots, a_{\mu}$ per assicurare che $m_{s}^{\prime}>m_{s}>\mu$. Allora

$$
0<F(x+h)-F(x)<u_{\beta+1}+u_{\mu+2}+\ldots
$$

e quindi, per la convergenza, della serie $S,|F(x+h)-F(x)|<\varepsilon$ assegnato arbitrariamente piccolo.

La $F(x)$ è continua verso sinistra in tutti i punti $x$ di $\overline{a b}$ che non appartengono ad $\mathcal{O}$. Infatti l'ineremento sinistro di $F(x)$ relativo ad $x$ (ora è $h<0$ ) è ancora della forma (3): il minuendo è una somma di un numero finito di termini se $x+h$ è un punto di $\mathscr{V}$ (ed eventualmente può scomparire), è una serie nel caso contrario; il sottraendo è sempre una serie. In ogni caso è

$$
a_{m s} \leq x+h<a_{m^{\prime}}<x
$$

Partendo da un valore iniziale facciamo tendere $h$ a 0 . Quando $x+h$ raggiunge il valore $a_{m^{\prime} s}$, il sottraendo perde il primo termine $u_{m^{\prime} s}$ e il minuendo si riduce a 0 . Quando $x+h$ sorpassa il valore $a_{m r^{\prime}}$, il sottraendo resta stazionario mentre il minuendo si riforma a cominciare da un termine con indice superiore ad $m_{s+1}^{\prime}$. Dunque è

$$
|F(x+h)-F(x)|<u_{m}{ }^{\prime}+u_{m u_{s}^{\prime}+1}+\ldots
$$

e, per la convergenza della serie $S,|F(x+h)-F(x)|<\varepsilon$ arbitrariamente asseguato.

Se $x$ appartiene ad $\mathcal{O}$ (sia precisamente, conservando la notazione, $\left.x \equiv a_{r^{\prime}} \equiv a_{m^{\prime} n}\right)$, il sottraendo nel secondo membro della (3) è sempre $\neq 0$, 
riducendosi ad $u_{m^{\prime} n}$ quando $h>x-a_{n t^{\prime}-1}$. Il minuendo, quando $h$ tende a zero, tende ad una serie ben determinata: è la serie dei termini $u$ corrispondenti al gruppo estratto da $\mathscr{T}$ con la legge descritta, relativamente all' intervallo $\overline{a_{m_{n-1}} x}$. La serie $S$ deve dunque essere scelta in modo che $F(x+1-h)-$ - $F(x)$ tenda a un valore $\neq 0$ quando $h$ tende a 0 .

In un lavoro in corso di stampa nei * Rendiconti del Circolo Matematico di Palermo . esporgo un esempio molto semplice di funzione continua verso destra definita secondo le regole del presente numero. 